\title{
RECHERCHES SUR LES TRÉMATODES DE LA PIE
}

\section{EN PROVENCE}

\author{
Par Jean TIMON-DAVID
}

Les recherches que je poursuis depuis quelques années sur les Helminthes de la Pie m'ont montré le grand intérêt qui s'attache à l'étude des Trématodes chez cet oiseau. En raison de sa grande fréquence sur notre territoire, de sa voracité et de la grande diversité de son régime, la Pie se contamine facilement, et constitue un matériel de choix pour les recherches parasitologiques. Aucune monographie n'a été consacrée à cette question dans notre pays, mais des données précieuses ont été apportées par R.-Ph. Dollfus sur les parasites de la Pie à Richelieu et en Camargue. A l'étranger, il faut mentionner le travail de S. Markowski (1933) sur les Helminthes des Corvidés en Pologne et celui de Strom (1940) sur les Trématodes de la Pie en Kirghizie.

La liste que j'ai dressée jusqu'à ce jour compte onze espèces, dont trois nouvelles (voir Bull. Soc. Zool. Fr., 1950, p. 243-246, et 1953, p. 504-510) ; elle est certainement loin d'être close et on doit s'attendre à d'autres découvertes chez des sujets tués dans des localités différentes et, particulièrement, en Camargue.

Le matériel qui fait l'objet de cette étude provient des environs d'Aix-en-Provence, commune du Tholonet (Bouches-du-Rhône). Les oiseaux ont été tués pendant la saison d'été, de juillet à septembre 1950-53. Trente-six sujets ont été examinés. Les dissections ont été faites aussitôt après la mort, dans un délai variant de quelques minutes à une heure. Les parasites étaient logés : dans les sacs aériens (une espèce); dans le tube digestif (une espèce); dans la bourse de Fabricius (deux espèces) ; dans les reins et les uretères (trois espèces); dans le foie et les voies biliaires (quatre espèces).

En dehors de la description des espèces nouvelles, l'abondance du matériel que j'ai recueilli m'a permis d'étudier l'amplitude des variations chez diverses formes où elle était mal connue. Des tableaux de mensurations précisent ces résultats et donnent les dimensions extrêmes atteintes par les divers organes.

Ann. de Parasitologie, T. XXViII, No $4 .-1953$. 
Les documents que j'ai rassemblés fournissent d'autre part des données nouvelles sur la biogéographie des Trématodes de France et sur la faunistique. Nos connaissances sont encore extrêmement lacunaires à cet égard : c’est ainsi que Tamerlania zarudnyi Skrjabin 1924 (= gallica Dollfus 1946), signalé seulement à Richelieu (Indre-et-Loire), est abondant chez la Pie au Tholonet ; Prosthogonimus brauni Skrjabin, considéré comme rare en France, est commun chez ce même hôte au Tholonet; Brachylecithum lobatum (Railliet) se rencontre avec une grande fréquence dans les canalicules biliaires.

L'observation des parasites in situ et le prélèvement de nombreuses pièces histologiques m'ont permis de faire une étude anatomopathologique des lésions qu'ils provoquent, particulièrement dans le cas des reins, des uretères et du foie. L'interprétation des coupes permet d'apprécier les réactions de I'hôte et donne des indications sur la biologie du parasite.

Je n’ai, naturellement, pas négligé d'orienter mes recherches vers la découverte des cycles évolutifs. Dans ce but, le contenu stomacal et intestinal des Pies a été soigneusement analysé et déterminé dans tous les cas où l'état de conservation le permettait.

J'ai entrepris la recherche des formes larvaires chez les Mollusques et les autres invertébrés de la région où ont eu lieu les chasses. Des résultats ont été obtenus en ce qui concerne les métacercaires de Brachylæmus, dont je donne des figures.

\section{Technigues}

Les viscères, découpés en fragments, ont été soigneusement passés en revue au binoculaire ; les vers ont été tués en extension dans de l'eau isotonique et fixés à l'alcool-formol. Les préparations totales ont été colorées au carmin boracique de Grenacher ; les pièces histologiques, fixées au Bouin, ont été coupées et colorées par les méthodes habituelles (hémalun-éosine; Van Gieson au jaune de métanile ; trichromiques de Masson à l'hémalun-érythrosine-safran et de Cajal au magenta-picro-carmin d'indigo).

\section{1. -- Trématodes des sacs aériens}

\section{Cyclocolum (Pseudhyptiasmus) Dollfusi Timon-David 1950}

J'ai décrit cette belle espèce en 1950 d'après 25 exemplaires trouvés dans les sacs aériens d'une Pie, au Tholonet; depuis, je l'ai retrouvée une seule fois ; c'est donc une forme assez rare : le pourcentage d'infestation correspond à 5,5 p. 100 . 
Dans le premier cas (9 septembre 1950), les parasites étaient groupés par amas de quatre ou cinq dans les sacs aériens thoraciques ; dans le second (30 aoùt 1952), ils étaient seulement au nombre de quatre en tout, au-dessus du rein gauche.

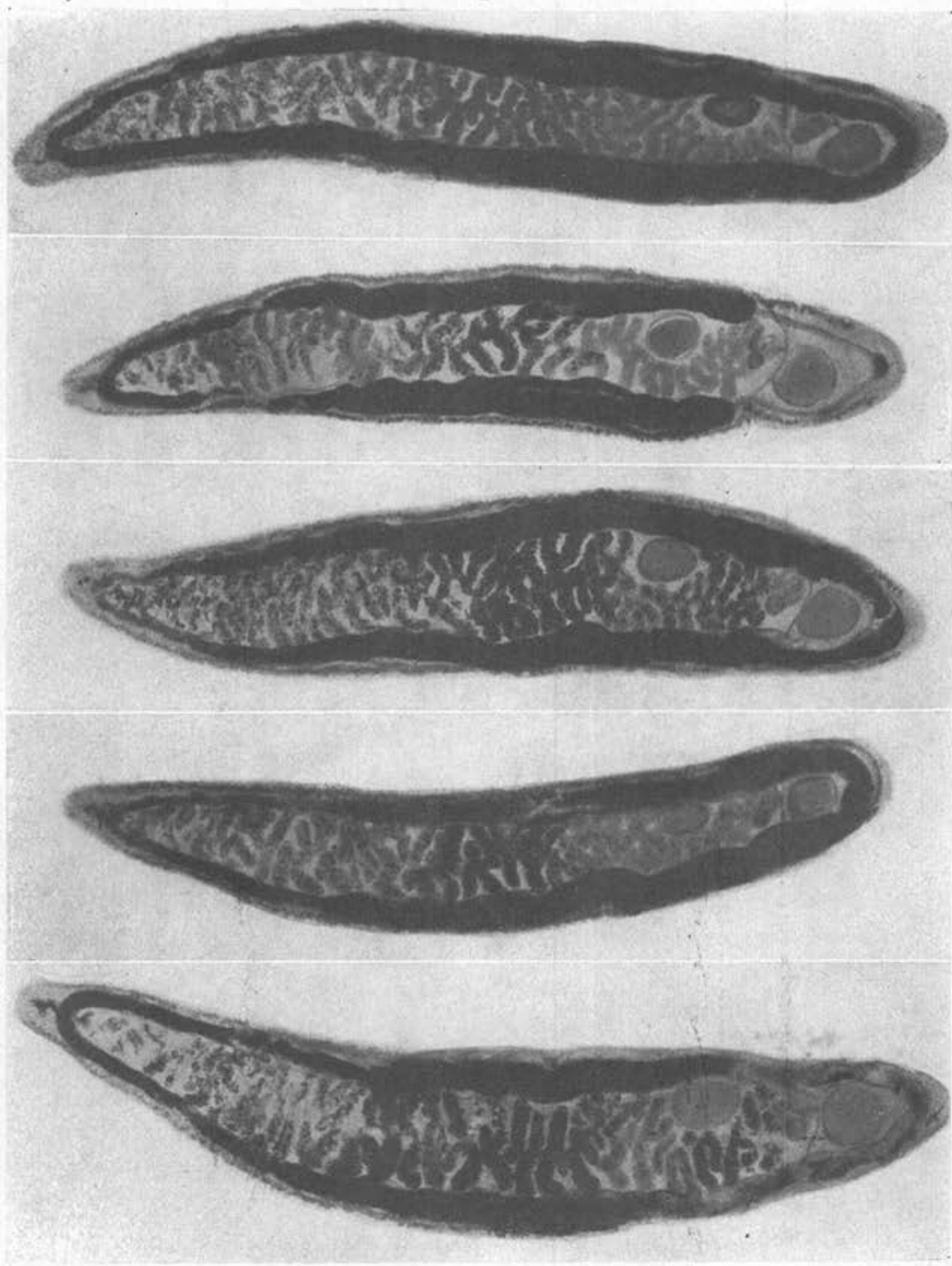

Fig. 1. - Cyclocolum (Pseudhyptiasmus) Dollfusi Timon-David 1950. Sacs aériens de la Pie. Microphotographies montrant les variations individuelles de cinq sujets provenant du même hôte (Color. au carmin boracique de Grenacher). 
Une étude plus détaillée me permet de compléter ma première description :

L'espèce est de grande taille, allongée, à bords subparallèles. Les sıjets les plus grands, en extension, mesurent $20 \mathrm{~mm} . \times 4 \mathrm{~mm}$. (fig. $\mathbf{1} i$ :
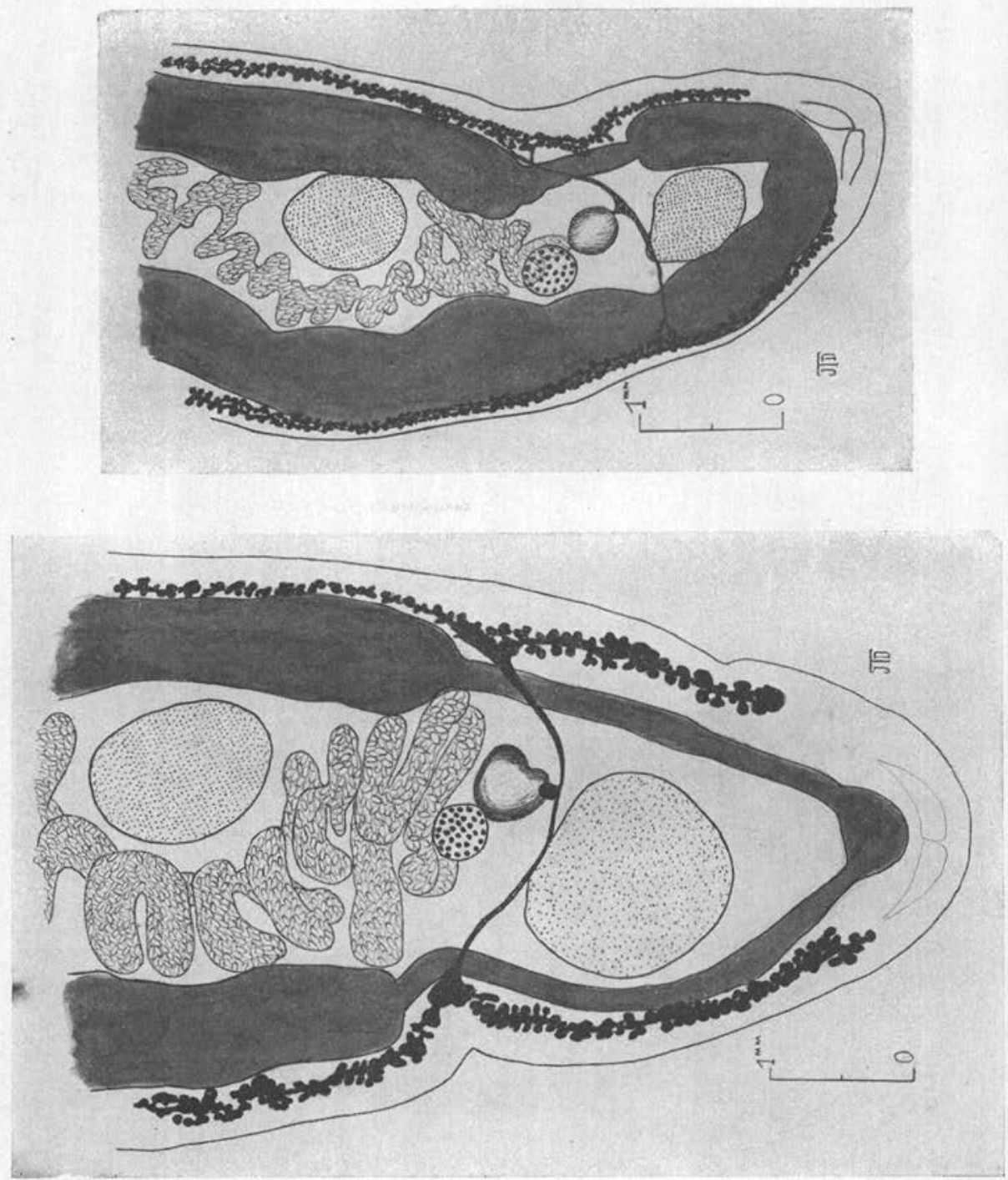

Fig. 2 et Fig. 3. - Cyclocœlum (Pseudhypticsmus) Dollfusi T.-D., région postérieure. On remarque la situation variable des constrictions cæcales, la disposition des vitellogènes et le trajet du vitelloducte transverse. 
les plus petits $15,5 \mathrm{~mm} . \times 2,9 \mathrm{~mm}$; les dimensions moyennes sont de $17 \mathrm{~mm}$. $\times 3,2 \mathrm{~mm}$. La ventouse orale est faible, presque dépourvue de musculature; son observation est assez difficile; son diamètre est compris entre 0,350 et $0,420 \mathrm{~mm}$. Il n'y a pas d'acétabulum. Le pharynx est robuste, globuleux (longueur 0,250 à $0,280 \mathrm{~mm}$; largeur 0,250 à $0,280 \mathrm{~mm}$.), précédé d'un très court prépharynx. L'œesophage mesure 0,250 à $0,425 \mathrm{~mm}$. Les cæca sont très apparents : leur contenu (sang ?) leur communique en général une teinte noirâtre qui rend leur observation très facile ; ils s'allongent parallèlement aux côtés du corps et s'anastomosent en anse près de l'extrémité postérieure; leur diamètre est très variable $(0,1$ à $1 \mathrm{~mm}$.) ; ils présentent des dilatations ampulaires séparées par des constrictions, plus ou moins étendues, qui peuvent siéger en des points très divers. Chez certains sujets (fig. 2), les branches intestinales se retrécissent brusquement au niveau de l'ovaire, l'anse terminale

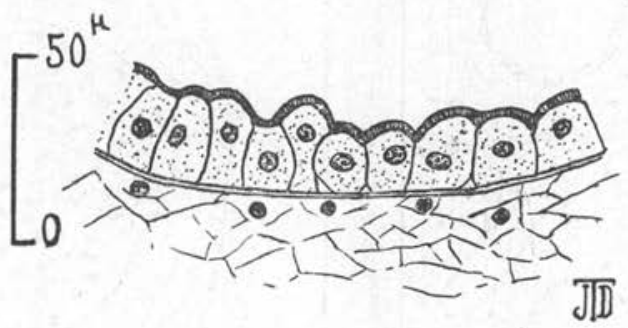

Fig. 4. - Cyclocalum (Pseudhyptiasmus) Dollfusi T.-D. Coupe dans la paroi d'un excum, montrant la structure histologique.

étant sensiblement plus étroite ; chez d'autres (fig. 3), cette anse est aussi large que les portions précédentes. Les constrictions peuvent aussi être situées en avant, d'un seul côté ou des deux à la fois.

Histologiquement, la paroi intestinale est constituée par un revêtement épithélial implanté sur une mince basale ; ces cellules sont arrondies ou cubiques; leur hauteur varie de 12 à $24 \mu$; leur bord apical est limité par un plateau strié (fig. 4). Sur les coupes colorées à l'hémalun, ce plateau apparait sous forme d'une bande réfringente, beaucoup plus foncée que le corps cellulaire et se détache nettement sur la lumière du cacum.

L'appareil excréteur comprend une vessie formée de deux poches accolées, situées en arrière de la bouche intestinale. Le pore excréteur est subterminal. Les canaux décrivent un trajet d'une extrême complication, constituant un réseau avec une multitude d'anastomoses. Sur une coupe transversale, à la hauteur du testicule postérieur (fig. 6) ces canaux sont au nombre de 26 ; leur calibre, très inégal, va de 30 à $150 \mu$. Il est facile de suivre leurs ramifications et anastomoses sur les coupes en série ; leur situation est périphérique, entourant tout le corps sous la couche musculaire interne ; latéralement, ils se développent en dehors des vitellogènes. 
La paroi de ces canaux est formée de cellules épithéliales très basses $(2,4$ à $4,8 \mu)$, dont les noyaux font souvent saillie dans la lumière du tube.

Le pore génital s'ouvre un peu en arrière du pharynx et non au niveau de son bord postérieur, comme je l'ai indiqué en 1950 ; cette disposition est très nette sur une coupe sagittale (fig. 7). Les testicules sont ovoïdes,
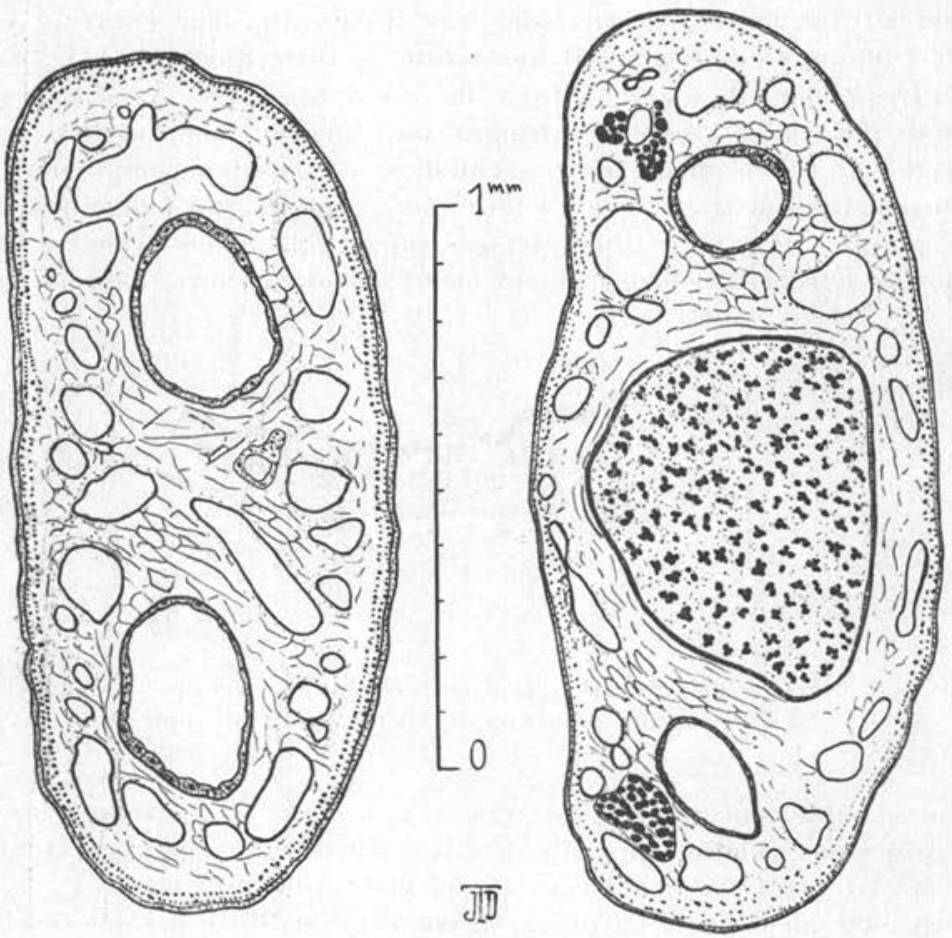

Fig. 5. - Cyclocolum (Pseudhyptiasmus) Dollfusi T.-D. Coupe transversale passant un peu en avant de la boucle intestinale. Le réseau des canaux excréteurs est bien visible.

Fig. 6. - Cyclocolum (Pseudhyptiasmus) Dollfusi T.-D. Coupe transversale au niveau du testicule postérieur.

très apparents, disposés l'un au-devant de l'autre dans la partie postérieure du corps ; leur grand axe mesure de 0,812 à $1,350 \mathrm{~mm}$.; le testicule antérieur est du côté gauche, accolé à la branche correspondante du tube digestif ; le postérieur du côté droit. La ligne qui joindait leurs centres est donc oblique par rapport à l'axe du corps. La poche du cirre, située en avant de la bifurcation du tube digestif, mesure en moyenne $0,250 \mathrm{~mm}$.

L'ovaire sphérique (diamètre $0,350 \mathrm{~mm}$. en moyenne) est entre les deux testicules, toujours plus rapproché du postérieur ; à côté de lui, immé- 
diatement en arrière, se trouve la glande de Mehlis $(0,5$ à $0,6 \mathrm{~mm}$.), entourée par la partie la plus concave du vitelloducte transverse. Je n'ai pas observé de receptaculum seminis.

L'extension des vitellogènes présente des variations individuelles assez importantes qu'il est nécessaire de préciser. Les follicules sont alignés de chaque côté, en dehors du cæcum, sur lequel ils peuvent empièter quand il est très large (fig. 3); leur limite antérieure peut atteindre la bifurcation du tube digestif ou s'avancer jusqu'au niveau du bord postérieur du pharynx; en arrière, ils peuvent se terminer à la hauteur du bord antérieur du testicule postérieur ou s'étendre sensiblement plus loin, jusqu'à l'extrémité de la boucle intestinale. A noter une dissymétrie fréquente chez un même individu: la différence d'extension des vitellogènes des deux côtés peut atteindre $0,30 \mathrm{~mm}$., aussi bien en avant qu'en arrière.

Le vitelloducte transverse, très facile à observer, se constitue aux dépens de plusieurs canaux qui convergent; il dessine une courbe à concavité antérieure très accusée, passant entre l'ovaire et le testicule postérieur ; son diamè-

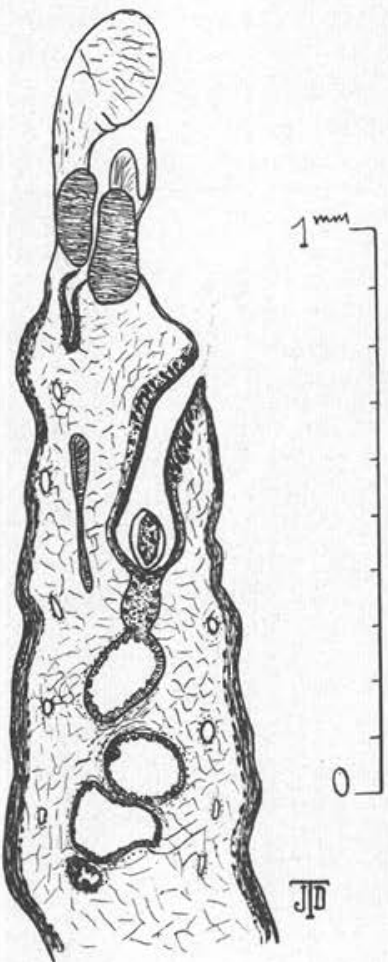

FIG. 7. - Cyclocxlum (Pseudhyptiasmus) Dollfusi T-D. Coupe sagitale montrant la position du pore génital. tre varie de 28 à $40 \mu$; le réservoir vitellin est ovoïde ou piriforme $(140 \mu \times 90 \mu)$.

Entre l'ootype et le pore génital se développent un grand nombre d'anses utérines orientées transversalement ou irrégulièrement, mais jamais récurrentes comme chez les Hyptiasminæ; elles n'empiètent jamais sur les cæca. Les dimensions les plus fréquentes des œufs correspondent à $127 \mu \times 66 \mu$ (voir le tableau). 


\begin{tabular}{|c|c|c|c|c|c|c|c|}
\hline 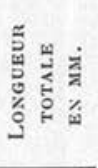 & 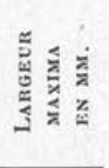 & 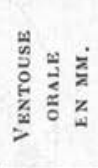 & 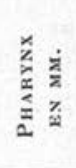 & 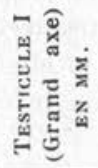 & 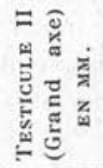 & 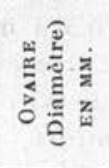 & $\begin{array}{l}0 \\
0 \\
0 \\
0 \\
0\end{array}$ \\
\hline 200 & 3 & 0,406 & $\begin{array}{l}0,252 \\
0252\end{array}$ & 1.232 & 1,428 & 0,392 & $127 \mu \times 72 \mu$ \\
\hline 145 & 2 & 0,380 & $\begin{array}{l}0,252 \\
0,252\end{array}$ & 0,812 & 0,770 & 0,378 & $120 \mu \times 60 \mu$ \\
\hline 169 & 2,6 & 0,410 & $\begin{array}{l}0,280 \\
0,266\end{array}$ & 0,952 & 1,260 & 0,356 & $127 \mu \times 72 \mu$ \\
\hline 161 & 3,2 & 0,350 & $\begin{array}{l}0,280 \\
0,280\end{array}$ & 1,092 & 1,176 & 0,350 & $127 \mu \times 72 \mu$ \\
\hline 165 & 3,2 & 0,420 & $\begin{array}{l}0,280 \\
0,266\end{array}$ & 1,120 & 1,162 & 0,364 & $122,4 \mu \times 62 \mu$ \\
\hline 159 & 2,8 & $0,4 i 0$ & $\begin{array}{l}0,210 \\
0,224\end{array}$ & 1,108 & 1,092 & 0,322 & $120 \mu \times 60 \mu$ \\
\hline 169 & 3,1 & 0,420 & $\begin{array}{l}0,280 \\
0,280\end{array}$ & 1,120 & 1,120 & 0,308 & $122 \mu \times 62 \mu$ \\
\hline 156 & 2,7 & 0,350 & $\begin{array}{l}0,280 \\
0,266\end{array}$ & 1.022 & 1,050 & 0,392 & $120 \mu \times 67 \mu$ \\
\hline 175 & 3,2 & 0,420 & $\begin{array}{l}0,224 \\
0,280\end{array}$ & 1,190 & 1,288 & 0,364 & $12 \bar{\mu} \mu \times 67,2 \mu$ \\
\hline 150 & 2,1 & 0,420 & $\begin{array}{l}0,280 \\
0,252\end{array}$ & 1,092 & 1,204 & 0,420 & $125 \mu \times 65 \mu$ \\
\hline
\end{tabular}

Mensurations de Cygclocalum (Pseudhyptiasmus) Dollfusi Timon-David 1950. 10 sujets bien développés, renfermant des æufs en abondance.

Discussion. - Dans sa mise au point de 1948, R.-Ph. Dollfus admet trois familles dans la superfamille des Cyclocoloidea :

Cyclocolidx: Pas de diverticules sur le bord interne de l'intestin ; ovaire jamais complètement et nettement en arrière du testicule postérieur, presque toujours plus ou moins loin en avant.

Bothriogastridæ: Pas de diverticules sur le bord interne de l'intestin ; ovaire toujours complètement et nettement en arrière du testicule postérieur.

Typhlocœlidæ: Bord interne de l'intestin pourvu d'une série de diverticules. 
C'est évidemment dans la première de ces familles que doit prendre place le parasite étudié ici. Les caractères considérés par R.-Ph. Dollfus comme les plus importants pour la définition des espèces résident dans la disposition des sinuosités utérines (orientées plus ou moins transversalement, irrégulièrement ou en direction postéro-externe) et dans la position des cæca, plus ou moins rapprochés des bords du corps. Il y a lieu de tenir compte ensuite de l'empiétement des anses utérines sur les branches intestinales et de la position relative des testicules et de l'ovaire.

Dans la disposition fondamentale des Cyclocolinæ, l'ovaire occupe le sommet d'un triangle, dont la base correspond à une ligne joignant les centres des deux testicules. Chez Pseudhyptiasmus Dollfus 1948, l'ovaire est situé entre les deux testicules, sur une même ligne droite, qui est plus ou moins oblique par rapport à l'axe du corps ; l'ovaire est beaucoup plus rapproché du testicule postérieur que de l'antérieur ; le pore génital est péranpharyngien ou opisthopharyngien. Ces caractères concordent parfaitement avec ceux que j'ai observés chez le parasite de la Pie.

Cyclocolum (Pseudhyptiasmus) Dollfusi Timon-David 1950 représente le premier Cyclocœlidé trouvé en France chez un Passériforme. Les espèces (peu nombreuses) signalées jusqu'ici dans notre pays proviennent d'Ansériformes, de Ralliformes et de Charadriiformes.

Le Cyclocoelum de la Pie n'est pas sans analogies avec C. (Pseudhyptiasmus) bivesiculatum S. Prudhoe 1944, trouvé chez Thereiceryx zeylanicus zeylanicus (Gmel.) à Ceylan, mais s'en distingue nettement (en dehors de l'hôte et de la localisation géographique) par sa taille beaucoup plus grande et par les dimensions relatives de ses organes : le rapport du diamètre de l'ovaire au grand axe du testicule est compris entre 0,29 et 0,35 chez $C$. Dollfusi, tandis qu'il atteint 0,5 à 1 chez $C$. bivesiculatum.

Cyclocœlum Dollfusi se distingue facilement de C. (Pseudhyptiasmus) ominosus (W. Kossack, 1911), dont la taille est un peu plus faible et les œufs beaucoup plus petits : 65 à $80 \mu$ (cavité thoracique de Grus grus L.). La différence est aussi très nette avec C. (Pseudhyptiasmus) vagum K. Morishita 1924, dont les œufs sont encore plus petits : 50 à $77 \mu$ (conduit lacrymo-nasal de Chrysolophus pictus L.). Chez Cyclocœelum (Pseudhyptiasmus) dumetellæ C. C. Zeliff 1943, la taille ne dépasse pas $8,5 \mathrm{~mm}$.; les vitellogènes s'étendent beaucoup moins en avant et la vessie n'est pas bilobée (sacs aériens de Dumetella carolinensis L.). Aucune confusion n'est possible avec C. (Pseudhyptiasmus) undulatum (W. P. N. Canavan, 1934), dont l'aspect général est très différent par suite de la disposition sinueuse 
des cæca, plus éloignés des bords du corps. La taille ne dépasse pas $11,5 \mathrm{~mm}$. La description de W.P. N. Canavan n'indique pas les dimensions des œufs (sacs aériens de Grus grus lilfordi Sharpe). C. (Pseudhyptiasmus) sinhaladvipa Fernando 1950 des sinus nasaux de Gallus lafayetti, à Cyelan, diffère de notre espèce à la fois par sa morphologie, par son hôte et par sa localisation géographique.

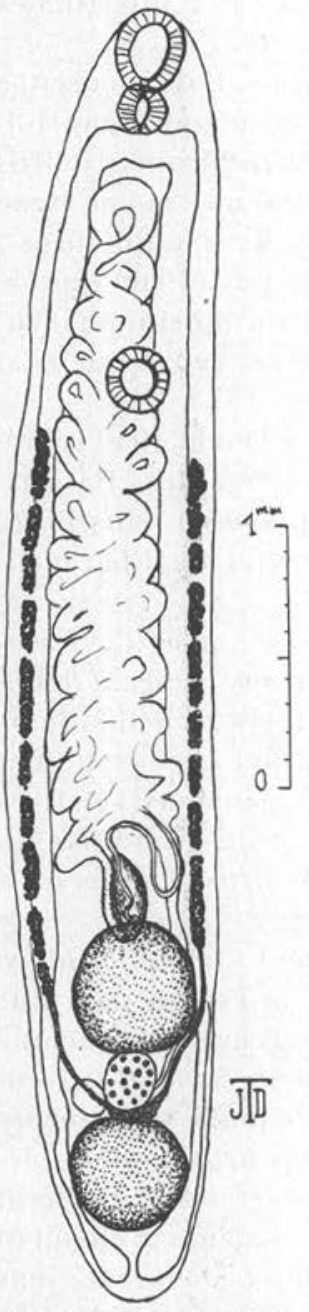

Fiti. 8. - Brachylamus fuscatus (Rud.). Intestin postérieur de Pie.

\section{II. - Trématodes du tube digestif}

\section{Brachylemus fuscatus (Rud.)}

Les Pies du Tholonet sont infestées dans la proportion de 20,8 p. 100 par un Brachylæmus Dujardin (Harmostomum Braun). J'ai trouvé ces Distomes en nombre variable (de 1 à 23) dans l'intestin, principalement dans les dix derniers centimètres.
Numéro d’ordre

$1010 \ldots \ldots \ldots \ldots$

$1046 \ldots . . . \ldots$.

$1048 \ldots \ldots \ldots$.

$1053 . \ldots \ldots \ldots$.

$1062 \ldots \ldots \ldots \ldots$

$1065 \ldots \ldots \ldots$.
Date

$8-9-1950$

$10-8-1952$

18-8-1952

27-8-1952

7-9-1952

8-9-1952
Nombre de Brachylamus recueillis

\begin{tabular}{r}
\hline 1 \\
5 \\
2 \\
4 \\
23 \\
2
\end{tabular}

Ce Brachylæmus de la Pie (fig. 8) présente les caractères suivants : Corps allongé, à côtés subparallèles. Longueur totale (chez des sujets renfermant des œufs en abondance) : $2,85 \mathrm{~mm}$. à $5,80 \mathrm{~mm}$. (moyenne sur 12 individus : 4,01 mm.). Largeur maxima : $0,490 \mathrm{~mm}$. à $0,770 \mathrm{~mm}$. Ventouse orale généralement un peu plus longue que large, à ouverture en fente longitudinale ; dimensions moyennes : $0,235 \times 0,227 \mathrm{~mm}$. Diamètre de l'acetabulum : 0,200 à $0,280 \mathrm{~mm}$. (moyenne : 0,227). Le rapport $\frac{v \text {. orale }}{\mathrm{v} \text {. ventrale }}$ est donc compris entre 1 et 1,03 . La distance moyenne entre les centres des deux ventouses est de $0,988 \mathrm{~mm}$. Pharynx: longueur, 0,196 mm.; largeur, $0,210 \mathrm{~mm}$. Les testicules sont ovales: leur grand 
axe mesure en moyenne $0,336 \mathrm{~mm}$. Ovaire sphérique ou subsphérique ; diamètre moyen : $0,176 \mathrm{~mm}$. Ootype entre l'ovaire et le testicule postérieur. Eufs : 24 à $28,8 \mu \times 12$ à $16 \mu$; en moyenne : $26 \mu \times 14,4 \mu$. Les

\begin{tabular}{|c|c|c|c|c|c|c|c|c|c|}
\hline 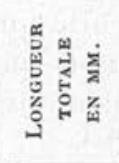 & 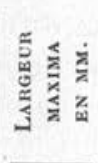 & 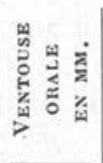 & 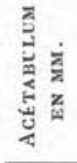 & 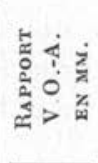 & 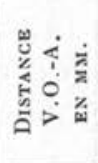 & 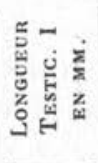 & 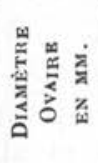 & 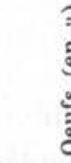 & है \\
\hline 3,30 & 0,670 & $\begin{array}{l}0,196 \\
0,196\end{array}$ & 0,210 & 0,94 & 1,050 & 0,295 & 0,168 & $\begin{array}{l}28,8 \\
14,4\end{array}$ & $x$ \\
\hline 3,36 & 0,532 & $\begin{array}{l}0, ? 24 \\
0,210\end{array}$ & 0,238 & 0,94 & 1,008 & 0,210 & 0,112 & $\begin{array}{l}28,6 \\
145\end{array}$ & $x$ \\
\hline 3,64 & 0,756 & $\begin{array}{l}0,252 \\
0,224\end{array}$ & 0,224 & $\begin{array}{c}1,1 \text { à } \\
1,0\end{array}$ & 0,840 & 0,322 & 0,196 & $\begin{array}{l}26,4 \\
14,4\end{array}$ & $x$ \\
\hline 4,06 & 0,504 & $\begin{array}{l}0,224 \\
0,224\end{array}$ & 0,224 & 1,0 & 0,980 & 0,350 & 0,182 & $\begin{array}{l}26,4 \\
14,4\end{array}$ & $x$ \\
\hline 4,214 & 0,770 & $\begin{array}{l}0,280 \\
0,252\end{array}$ & 0,280 & 1,0 & 1,050 & 0,378 & 0,168 & $\begin{array}{l}28,8 \\
16,8\end{array}$ & $x$ \\
\hline 3,360 & 0,602 & $\begin{array}{l}0,210 \\
0,210\end{array}$ & 0.210 & 1,0 & 0,952 & 0,322 & 0,168 & $\begin{array}{l}26,4 \\
14,4\end{array}$ & $x$ \\
\hline 4,088 & 0,630 & $\begin{array}{l}0,224 \\
0,196\end{array}$ & 0,210 & $\begin{array}{c}0,93 \text { à } \\
1,07\end{array}$ & $\mathrm{c}, 750$ & 0,308 & 0,182 & $\begin{array}{l}24,0 \\
14,0\end{array}$ & $x$ \\
\hline 2,856 & 0,490 & $\begin{array}{l}0,182 \\
0,182\end{array}$ & 0,200 & 0,91 & 0,672 & 0,322 & 0,168 & $\begin{array}{l}24,0 \\
14,4\end{array}$ & $x$ \\
\hline 3,70 & 0,700 & $\begin{array}{l}0,280 \\
0,252\end{array}$ & 0,224 & $\begin{array}{l}1,1 \text { à } \\
1,2\end{array}$ & $0,\{82$ & 0,252 & 0,168 & $\begin{array}{l}26,4 \\
14,4\end{array}$ & $x$ \\
\hline 5,80 & 0,672 & $\begin{array}{l}0.294 \\
0,280\end{array}$ & 0,280 & $\begin{array}{c}1,0 \text { à } \\
1,05\end{array}$ & 1,344 & 0,490 & 0,210 & $\begin{array}{l}24,0 \\
12,0\end{array}$ & $x$ \\
\hline 4,970 & 0,770 & $\begin{array}{l}0,238 \\
0,252\end{array}$ & 0,238 & $\begin{array}{c}1,0 \text { à } \\
1,05\end{array}$ & 1,260 & 0,462 & 0,210 & $\begin{array}{l}26,4 \\
16,8\end{array}$ & $x$ \\
\hline 4,46 & 0,500 & $\begin{array}{l}0,224 \\
0,224\end{array}$ & 0,224 & 1,0 & 1,078 & 0,322 & 0,182 & $\begin{array}{l}24,0 \\
14,0\end{array}$ & $\times$ \\
\hline $\begin{array}{c}2,45 \\
\text { (ceufspeu } \\
\text { nombr.) }\end{array}$ & 0,518 & $\begin{array}{l}0,266 \\
0,224\end{array}$ & 0,224 & $\begin{array}{c}1,0 \text { à } \\
1,17\end{array}$ & 0,658 & 0,294 & 0,168 & $\begin{array}{l}26,4 \\
14,4\end{array}$ & $\times$ \\
\hline
\end{tabular}

Mensurations du Brachylæmus de la Pie.

12 sujets renfermant des œufs mûrs en abondance ; chez le dernier, les cufs sont encore peu nombreux.

AnN. de Parasitologie, $\mathrm{T} . \mathrm{XXVIII}, \mathrm{N}^{\circ} 4 .-1953$. 
vitellogènes débutent un peu en arrière de l'acetabulum et s'étendent jusqu'à un point variable compris entre le bord et le premier tiers du testicule antérieur. Les téguments sont spinulés, surtout dans la partie antérieure du corps.

Beaucoup d'auteurs ont insisté depuis Braun sur la difficulté qui s'attache à la détermination des espèces du genre Brachylæmus par suite de leur grande uniformité morphologique et de l'ampleur des variations individuelles.

\section{Brachylæmus des Corvidés}

Aucun Brachylæmus ne paraìt avoir été signalé jusqu'ici chez la Pie en France; Dollfus (1934-35) n'en fait pas mention. Strom (1940) a trouvé deux espèces chez la Pie en U.R.S.S. : B. mesostomus (Rud.) et B. vastus n. sp.

Par contre, des Brachylæmus ont été souvent signalés chez d'autres Corvidés, dans notre pays et à l'étranger : B. fuscatus (Rud.) chez Corvus cornix L. en Pologne (Markowski, 1933) ; B. arcuatus (Duj.) chez Garrulus glandarius L. à Rennes (Dujardin, 1845), à Fribourg-en-Brisgau (Haussmann, 1899), à Richelieu (Indre-etLoire) (Dollfus, 1934), chez Corvus corone L. à Fribourg-en-Brisgau (Haussmann, 1899) ; B. caryocatactes (Zeder, 1800) chez Nucifraga caryocatactes (L.) (Allemagne et Musée de Vienne) et chez Graculus graculus (L.) (Musée de Vienne) (Diesing, 1850 ; Braun, 1902) ; B. mesostomus (Rud.) chez Coloeus monedula (L.) à Novotcherkassk et chez Trypanocorax frugilegus (L.) à Taschkent (Witenberg, 1925).

Discussion. - Parmi les Brachylæmus d'oiseaux, celui de la Pie ne saurait être confondu avec commutatus (Dies.) et sa variété gallinus (Witenb.), des Galliformes domestiques de la région paléarctique (sous-genre Postharmostomum Witenb., non retenu) (ouverture orale arrondie).

Il faut éliminer aussi les espèces brésiliennes : marsupium Braun, mordens Braun, centrodes Braun et mazzantii Travassos, chez lesquelles les vitellogènes s'étendent en avant de l'acétabulum ( $2^{\circ}$ groupe de Witenberg).

La discussion ne peut porter que sur l'attribution à l'une des espèces des Passériformes paléarctiques: caryocatactis (Zeder), mesostomus (Rud.), arcuatus (Dujardin), nicolli (Witenberg), inflatocolum Witenb. et fuscatus (Rud.). 
Chez B. caryocatactis (Zeder), les vitellogènes dépassent le bord postérieur de l'acétabulum et les œufs sont plus grands.

B. mesostomus (Rud.) est à éliminer en raison de sa petite taille (1 à $2 \mathrm{~mm}$.) et de l'aspect du corps qui est piriforme.

B. arcuatus (Dujardin), le « Brachylaime du Geai », que Witenberg avait considéré bien à tort comme une espèce douteuse, a été soigneusement redécrit par Dollfus (1934). Il a été trouvé six fois à Rennes sur 19 Geais et revu par Haussmann à Bâle et à Fribourgen-Brisgau ; Kurt Wolffhügel (1900) en a trouvé 70 exemplaires chez un seul Corvus corone L. A Richelieu, Dollfus l'a trouvé cinq fois chez le Geai (jusqu'à 30 exemplaires).

Cette espèce est évidemment très voisine de celle que j'ai trouvée chez la Pie; je ne crois pas, cependant, qu'on puisse identifier les deux formes : chez $B$. arcuatus (Duj.), les vitellogènes s'étendent plus loin en avant, atteignant le bord antérieur ou au moins le milieu de l'acétabulum; aucun de mes échantillons n'a présenté ce caractère. En outre, les œufs de l'espèce de Dujardin sont plus grands $(33 \mu \times 20 \mu$ en moyenne d'après Dollfus, au lieu de $26 \mu \times$ $14,4 \mu)$.

$B$. inflatocolum Witenb. est vraisemblablement synonyme de B. fuscatus, comme l'a indiqué Sinitsin (1931); nicolli Witenb. doit être rattaché à la même espèce (Joyeux, Baer et Timon-David, 1934).

J'admettrai done que le Brachylæmus de la Pie du Tholonet doit être déterminé $B$. fuscatus (Rud.) ; e'est l'espèce qui se trouve en abondance aux environs de Marseille chez le Moineau (Joyeux, Baer et Timon-David). Il y a cependant quelques légères différences, dues sans doute au changement d'hôte ; certains exemplaires du Brachylæmus de la Pie atteignent une taille un peu supérieure à celle de la forme du Moineau : jusqu'à $5,8 \mathrm{~mm}$. (contre 4 ); le diamètre de l'acétabulum peut atteindre $0,280 \mathrm{~mm}$. (contre 0,220 ). Les œufs sont sensiblement de la même taille.

\section{Formes Larvaires de Brachylæmus}

J'ai trouvé, à diverses reprises, des métacercaires de Brachylæmus dans les viscères d'Hélicidés (Helicopsis arenosa (Ziegler) Rossmässler 1838 (1), recueillis dans les champs fréquentés par les Pies au Tholonet. Il est évident qu'il s'agit bien de formes larvaires correspondant aux adultes hébergés par les oiseaux; ceux-ci se

(1) Je remercie très vivement $\mathrm{M}$. Cherbonnier qui a eu la grande obligeance d'examiner ces Helicopsis au Laboratoire de Malacologie du Muséum et à qui je dois cette précision. 
contaminent facilement en mangeant des Helicopsis que j'ai retrouvés dans leur estomac avec les métacercaires.

Je figure ici (fig. 9 et 10) deux exemplaires de ces métacercaires extraites de reins d'Helicopsis; l'un des sujets (fig. 10) est un peu contracté ; leur longueur est voisine de $0,600 \mathrm{~mm}$. ; leur largeur de $0,300 \mathrm{~mm}$. Ventouse orale : $0,180 \mathrm{~mm}$.; acétabulum : $0,145 \mathrm{~mm}$.; pharynx : $0,082 \times 0,080 \mathrm{~mm}$. Les cæca, après s'être détachés du pharynx, s'incurvent vers la ventouse orale, puis reviennent en
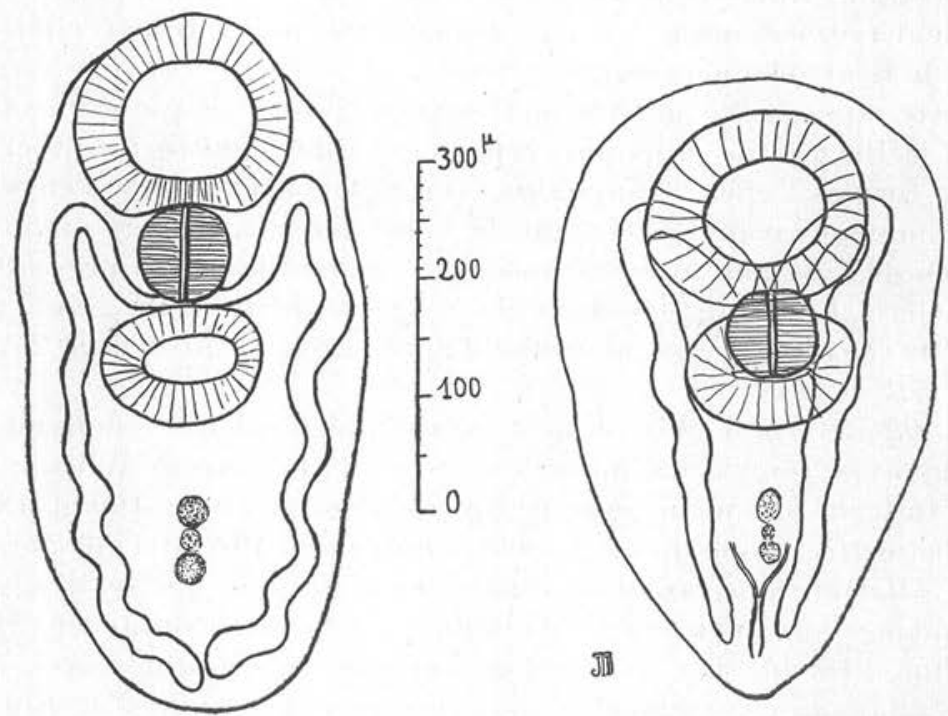

Fig. 9 et Fig. 10. - Métacercaire de Brachylæmus fuscatus (Rud.) extraite d'Helicopsis arenosa (Ziegler)

arrière en décrivant une boucle très accusée ; ils atteignent presque l'extrémité du corps. Les organes génitaux sont représentés par des ébauches qui fixent les colorants : une tache médiane correspond à l'ovaire entre les deux testicules. La vessie et les deux trones principaux sont bien visibles.

Je ne reviendrai pas ici sur la biologie des formes larvaires qui a été décrite chez divers Hélicidés récoltés aux environs de Marseille (Ch. Joyeux, J.-G. Baer et J. Timon-David, 1934); elle comporte des migrations chez le Mollusque et des contaminations entre individus vivant rassemblés en très grand nombre.

Des formes larvaires de Brachylæmus ont été signalées chez une série de Pulmonés terrestres ; voir notamment à ce sujet : W. Adam et E. Leloup (1934) et R.-Ph. Dollfus. (1934-1935). 


\section{III. - Trématodes de la Bourse de Fabricius}

J'ai recueilli 15 exemplaires de Prosthogonimus dans la bourse de Fabricius des Pies, au Tholonet; ce matériel a été fourni par cinq oiseaux qui en renfermaient de un à cinq.

Le genre Prosthogonimus Max Lühe est le type de la sous-famille des Prosthogoniminæ, parfois élevée au rang de famille (Lahille, 1922), dans la grande super-famille des Lepodermatoidea.

C'est seulement en 1948 que la présence de Prosthogonimus a été signalée en France pour la première fois par R.-Ph. Dollfus. Il est certain, cependant, que la prosthogonimose des oiseaux de bassecour sévit depuis longtemps dans notre pays, où elle doit être entretenue par l'infestation des oiseaux sauvages. Mes recherches montrent que la Pie doit être regardée à cet égard comme un hôte dangereux, fréquemment contaminé en Provence; son écologie, ses habitudes, qui le portent à fréquenter le voisinage des fermes et des élevages, doivent faire considérer cet oiseau comme un « réservoir de virus » de la prosthogonimose. Cette découverte présente un certain intérêt, car les Prosthogonimus n'avaient été signalés jusqu'ici en France, chez la Pie, qu'à titre de grandes raretés. La monographie de R.-Ph. Dollfus (1948) cite seulement deux observations : un exemplaire unique, recueilli par l'auteur lui-même à Richelieu (Indre-et-Loire), attribué provisoirement à Prosthogonimus brauni Skrjabin, et un autre trouvé par C. Desportes en Camargue et déterminé $P$. ovatus (Rud.).

En revanche, à l'étranger, $P$. ovatus (Rud.) a été signalé à diverses reprises chez la Pie en Allemagne, en Autriche et en U.R.S.S. ; P. cuneatus (Rud.) a été trouvé en Pologne et en U.R.S.S. ; il faut ajouter diverses mentions de Prosthogonimus indéterminés.

Le cycle évolutif des Prosthogonimus est connu grâce aux recherches d'un certain nombre d'auteurs et, en particulier, de R. W. Macy (1934-1939). Le Mollusque premier hôte a été identifié dans le cas de $P$. macrorchis R. W. Macy : c'est un Gastéropode aquatique du genre Amnicola; le second hôte est un Insecte, le plus souvent un Odonate. La métacercaire enkystée a été trouvée chez diverses espèces (larves, nymphes et imagos), dans la musculature abdominale ; l'infestation expérimentale d'oiseaux a été souvent réalisée à partir d'Odonates (voir R.-Ph. Dollfus, 1948, p. 7).

Sur les 15 exemplaires de Prosthogonimus que j'ai recueillis chez la Pie, 14 me paraissent appartenir à une même espèce ( $P$. aff. Brauni Skrjabin) (fig. 11); un seul à P. cuneatus (Rud.) (fig. 12). 


\section{Prosthogonimus aff. Brauni (Skrjabin 1919)}

Mes échantillons présentent les caractères suivants: Longueur en extension, 5 à $8 \mathrm{~mm}$. ; largeur maxima, 3 à 4,5 mm. Ventouse orale, 0,330 à $0,546 \mathrm{~mm}$. Acetabulum, 0,770 à $0,980 \mathrm{~mm}$. Le rapport des diamètres $\frac{\text { acetabulum }}{\text { ventouse orale }}$ est, dans la plupart des cas, voisin de 1,8 ; il s'élève cependant à 2,5 chez un sujet. L'ovaire est nettement post-acétabulaire, séparé de la bifurcation intestinale par une distance en général égale à son diamètre, parfois inférieure. La poche du cirre est sinueuse; les vitellogènes, disposés en grappes, s'étendent postérieurement au delà des testicules et dépassent franchement l'acetabulum en avant. L'utérus se développe de chaque côté largement au dehors des cæca intestinaux. Les œufs mûrs mesurent 22 à $24 \mu \times 12$ à $13 \mu$.

Ce Prosthogonimus (fig. 11) me parait identique à l'exemplaire unique décrit par R.-Ph. Dollfus (1948) chez la Pie à Richelieu et attribué avec quelque doute à $P$. Brauni Skrjabin. Comme la forme de Richelieu, mes exemplaires ne concordent pas exactement avec la figure de J. K. Skrjabin : l'acétabulum est moins rapproché de la bifurcation intestinale ; les vitellogènes s'étendent plus loin en avant et en arrière ; la poche du cirre contournée n'atteint pas le bord de l'acétabulum.

\begin{tabular}{|c|c|c|c|c|c|c|c|c|}
\hline 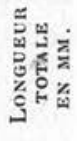 & 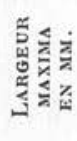 & 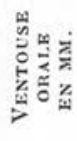 & 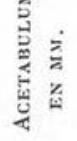 & \begin{tabular}{l|l} 
& \\
&
\end{tabular} & 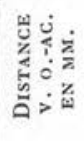 & 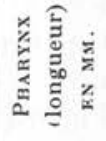 & 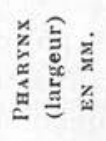 & $\frac{5}{0}$ \\
\hline 8 & 1,5 & 0,460 & 0,790 & 1,7 & 2,3 & 0,269 & 0,222 & $24 \mu \times 12 \mu$ \\
\hline 7,9 & 4,1 & 0,462 & 0,840 & 1,8 & 2,2 & 0,210 & 0,210 & $24 \times 13 \mu$ \\
\hline 6,5 & 4,0 & 0,490 & 0,826 & 1,7 & 2,2 & 0,224 & 0,182 & $24 \times 13 \mu$ \\
\hline 5 & 3,2 & 0,330 & 0,840 & 2,5 & 1,49 & 0.210 & 0,210 & $24 \times 13 \mu$ \\
\hline 5,7 & 3,0 & 0,434 & 0,770 & 1,7 & 2,13 & 0,224 & 0,238 & $21 \times 12 \mu$ \\
\hline 6,7 & 3,0 & 0,462 & 0840 & 1,8 & 2,57 & 0,210 & 0,238 & $24 \times 12 \mu$ \\
\hline 5,5 & 3,2 & 0,546 & 0,980 & 1,8 & 2,45 & 0,252 & 0,266 & $22 \times 13 \mu$ \\
\hline 6,2 & 3,5 & 0,490 & 0,910 & 1,8 & 2,177 & 0,224 & 0,238 & $24 \times 12 \mu$ \\
\hline 7,8 & 3,2 & 0,392 & 0,924 & 2,3 & 2,77 & 0,210 & 0,280 & $22 \times 12 \mu$ \\
\hline
\end{tabular}

Mensurations de 9 exemplaires de Prosthogonimus aff. Brauni Skrjabin. 


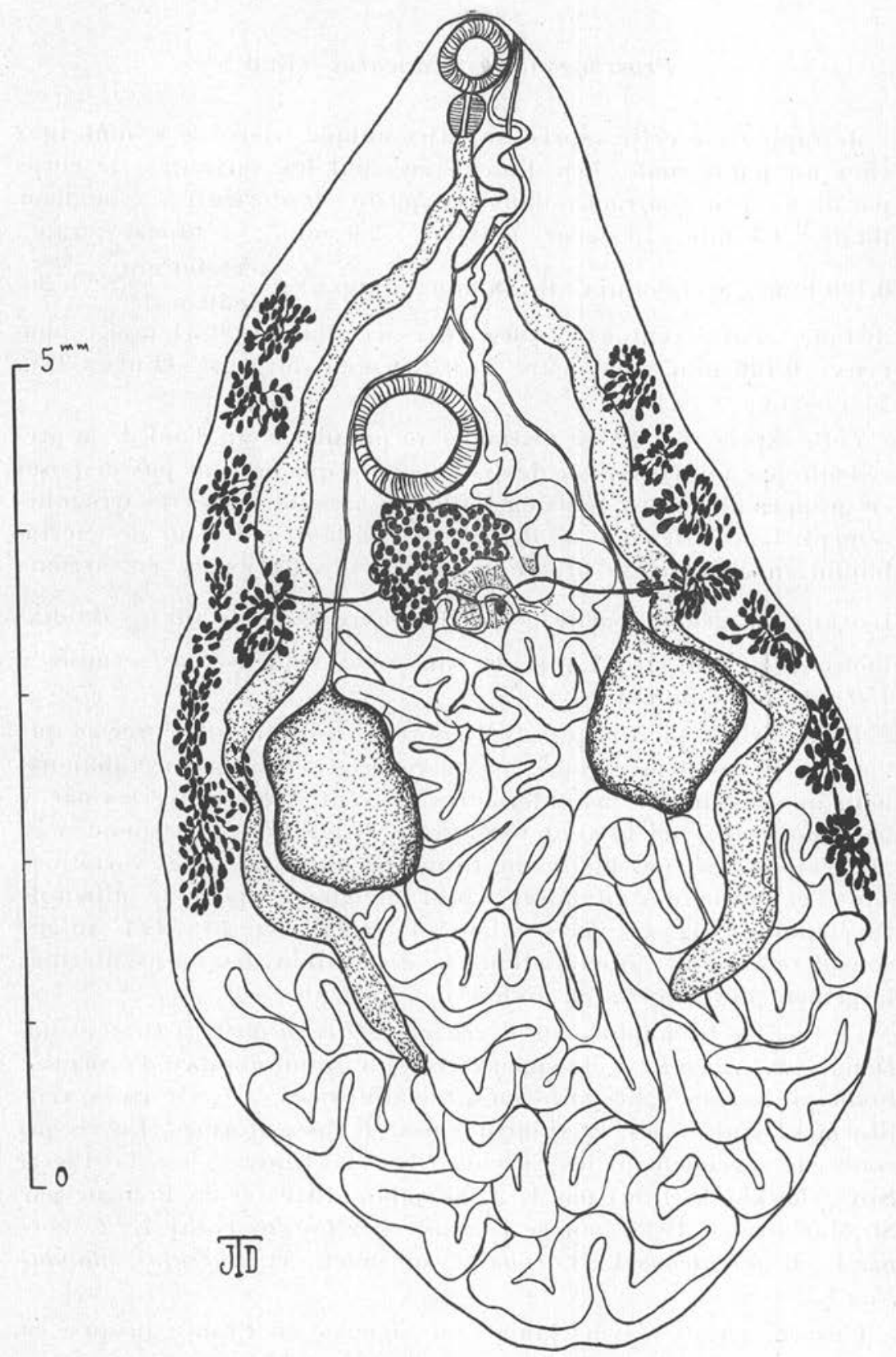

Fig. 11. - Prosthogonimus aff. Brauni K. I. Skrjabin. Bourse de Fabricius de la Pie. 


\section{Prosthogonimus cuneatus (Rud.)}

Je rapporte à cette espèce un sujet unique trouvé le 8 août 1952 chez un jeune mâle. Les dimensions sont les suivantes (le corps parait un peu contracté dans sa partie postérieure): longueur totale : 4,5 mm.; largeur maxima : $2,6 \mathrm{~mm}$.; ventouse orale : $0,406 \mathrm{~mm}$. ; acétabulum : 0,728 mm. ; rapport $\frac{\text { acetabulum }}{\text { vent. orale }}: 1,79$; distance centre ventouse orale-centre acétabulum : $1,54 \mathrm{~mm}$. ; pharynx : $0,196 \mathrm{~mm}$. (longueur) $\times 0,210 \mathrm{~mm}$. (largeur). CEufs : 22 à $24 \mu \times 13 \mu$.

Cette espèce (fig. 12) se distingue au premier coup d'œil de la précédente par la disposition des vitellogènes qui ne sont pas disposés en grappes distinctes, mais en follicules arrondis et serrés irrégulièrement. Leur extension ne dépasse pas le bord antérieur de l'acétabulum en avant et se prolonge au delà des testicules en arrière. L'ovaire, en grande partie postacétabulaire, empiète sur $\frac{1}{8}$ du diamètre de la ventouse. La poche du cirre, sinueuse, se termine à $150 \mu$ de l'acétabulum.

Braun (1902, p. 76), qui a revu le type de Rudolphi, précise que l'ovaire, profondément lobé, est en règle générale postacétabulaire, mais que ses digitations antérieures peuvent être recouvertes par le bord postérieur de la ventouse : cette disposition correspond à ce que j'ai observé. Les figures de Braun accusent aussi des variations dans l'extension des vitellogènes, qui atteignent à peine le milieu de l'acétabulum (fig. 44) ou son bord antérieur (fig. 45). Cet auteur considère comme caractéristique la disposition des anses utérines largement développées en arrière des testicules.

Le type de Rudolphi a été décrit chez Otis tarda L. à Greifswald. Depuis, $P$. cuneatus a été signalé chez un grand nombre d'oiseaux : Podicipitiformes, Pélécaniformes, Ciconiiformes, Ansériformes, Galliformes, Gruiformes, Charadriiformes et Passériformes. En ce qui concerne spécialement les Corvidæ, il a été trouvé chez la Pie à Novotcherkassk (Don) par K. J. Skrjabin (1919) et en Pologne par St. Markowski (1933) ; on le connait chez Corvus corax L., C. cornix L., C. frugilegus L., Corvus pl. sp. indét., et Garrulus glandarius $\mathrm{L}$.

L'espèce paraît n'avoir jamais été signalée en France jusqu'à ce jour. 


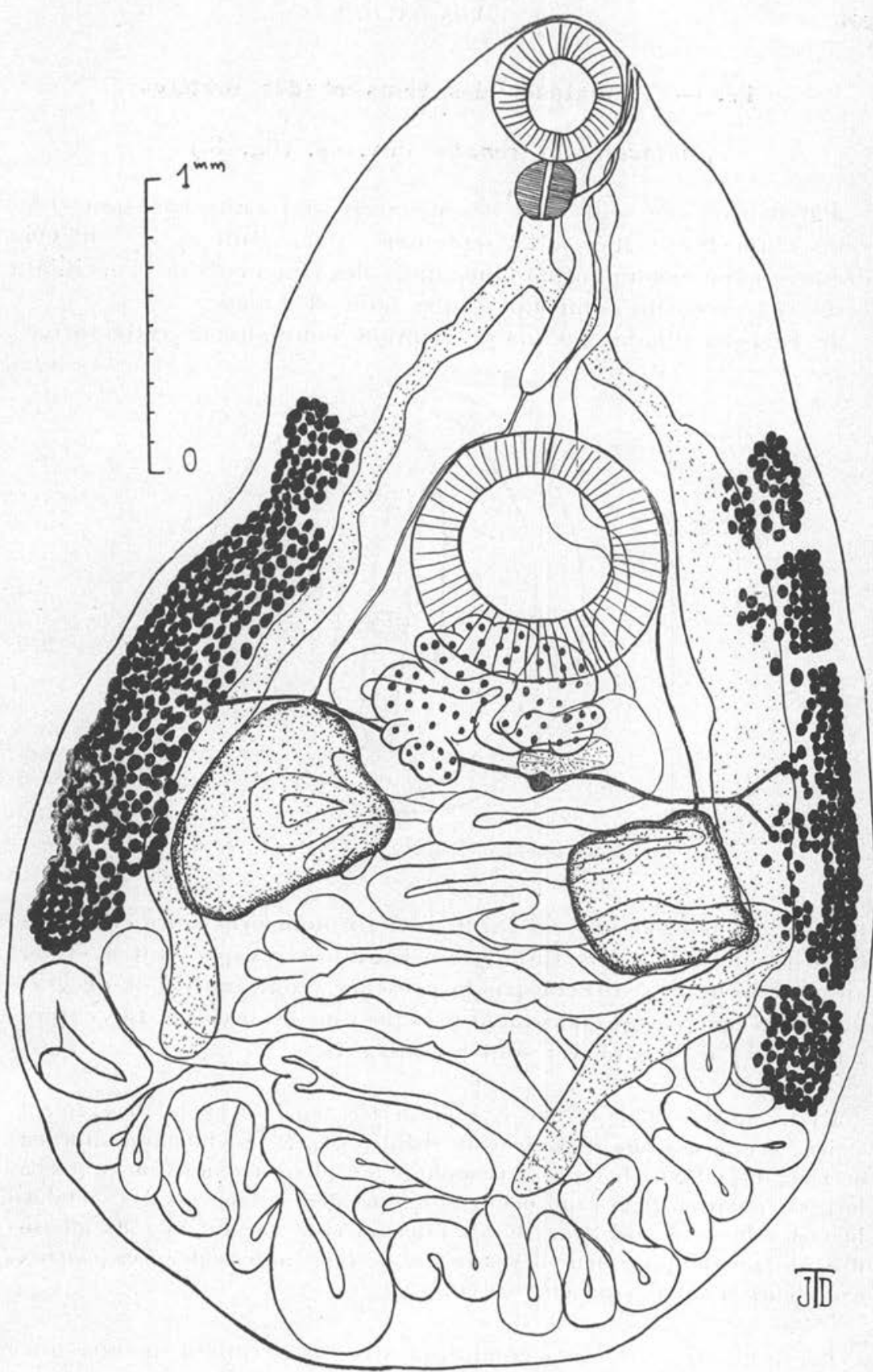

Fic. 12. - Prosthogonimus cuneatus (Rud.). Bourse de Fabricius de la Pie. 


\section{IV. - Trématodes des reins et des uretêres}

Metacercaria renalis nov. sp. (fig. 13)

J'ai trouvé une seule fois des métacercaires enkystées dans les reins d'une Pie ( $q$ tuée le 26 septembre 1952). Huit kystes ont été découverts en examinant au binoculaire des fragments de reins dilacérés et légèrement comprimés entre lame et lamelle.

Je ne peux donner qu'une description sommaire de cette forme

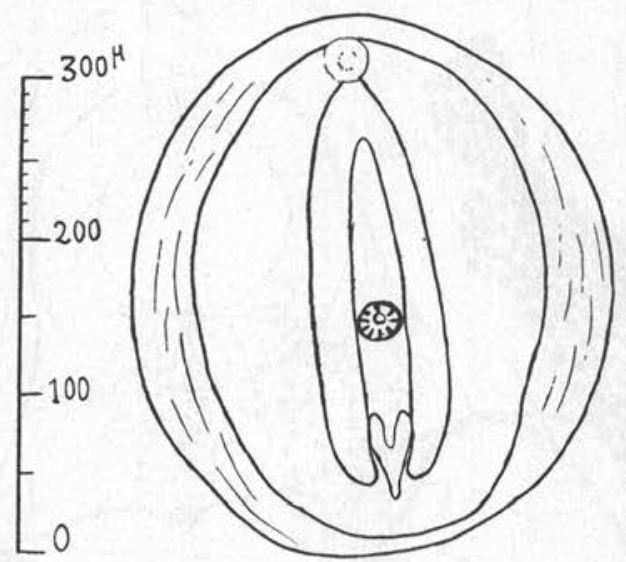

F1G. 13. - Metacercaria renalis nov. sp. Rein de Pie.

larvaire, et je crois utile de lui donner un nom provisoire en attendant qu'elle puisse être rattachée à l'adulte correspondant. L'étude en est rendue très difficile par la présence d'une multitude de granulations opaques qui masquent presque tous les organes. Les caractères que j'ai pu observer sont les suivants :

Kystes ovales $(330 \times 300 \mu)$, à paroi très épaisse $(40 \mu)$, légèrement striée. Ventouse orale difficilement visible $(28 \mu$ ?). Acetabulum situé en arrière du milieu du corps. Esophage et pharynx indistincts. Cæca larges d'environ $28 \mu$, rapprochés et subparallèles, tangents à l'acetabulum et à la vessie par leurs bords internes et se terminant sans atteindre l'extrémité postérieure. Vessie en $\mathrm{Y}$, bien apparente. Les canaux excréteurs n'ont pas pu être observés.

La rareté de cette métacercaire ne m'a pas permis d'en faire une étude plus poussée, ni de préciser sa localisation anatomique dans le rein. 
L'hôte définitif est à rechercher parmi les Falconiformes qui sont communs dans la région (Chaîne de Sainte-Victoire).

\section{Tamerlania zarudnyi (K. I. Skrjabin)}

Cinq Pies m'ont fourni des exemplaires d'un Tamerlania (fig. 14) que je détermine $T$. zarudnyi Skrjab. Le nombre des sujets variait de trois à une quarantaine par oiseau; ils étaient logés dans les canaux urinifères et dans l'uretère.

Les mensurations que j'ai relevées chez quelques individus sont groupées dans le tableau suivant :

\begin{tabular}{|c|c|c|c|c|c|c|}
\hline 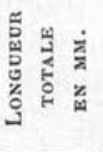 & 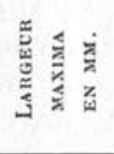 & 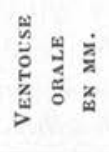 & 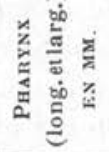 & 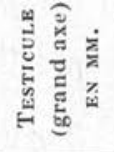 & 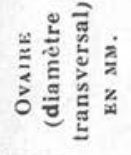 & $\frac{n}{\dot{\theta}}$ \\
\hline 3,18 & 0,7 & 0,224 & $\begin{array}{l}0,112 \\
0,112\end{array}$ & 0,280 & 0,182 & $38,4 \times 19,2 \mu$ \\
\hline 2,56 & 0,56 & 0,266 & $\begin{array}{l}0,085 \\
0,098\end{array}$ & 0,210 & 0,140 & $\begin{array}{l}33.6 \times 24 \mu \\
38,4 \times 24\end{array}$ \\
\hline 3,92 & 0,58 & 0,280 & $\begin{array}{l}0,112 \\
0,126\end{array}$ & 0,266 & 0,154 & $38,4 \times 21 \mu$ \\
\hline 3,08 & 0,770 & 0,280 & $\begin{array}{l}0,112 \\
0,126\end{array}$ & 0,224 & 0,168 & $36 \times 21,6 \mu$ \\
\hline 3,36 & 0,602 & 0,252 & $\begin{array}{l}0,112 \\
0,140\end{array}$ & 0,224 & 0,182 & $36 \times 21,6 \mu$ \\
\hline 3,58 & 0,630 & 0,280 & $\begin{array}{l}0,120 \\
0,125\end{array}$ & 0,280 & 0.168 & $38,4 \times 21,6 \mu$ \\
\hline 2,77 & 0,812 & 0,280 & $\begin{array}{l}0,112 \\
0,140\end{array}$ & 0,280 & 0,224 & $38,4 \times 21,6 \mu$ \\
\hline
\end{tabular}

Le genre Tamerlania (famille des Eucotylidæx) a été proposé en 1924 par K. I. Skrjabin pour un parasite découvert dans les canaux urinifères de Passer'montanus, au Turkestan russe. D'autres espèces ont été décrites ultérieurement: T. meruli N. A. Nezlobinsky 1926 chez Turdus merula L. ; T. japonica S. Yamaguti 1935 chez Coccothraustes coccothraustes japonicus Temm. et Schlegel ; T. bragai V. Dos Santos 1934 chez Columba livia Gmel. domestica ; T. melospizæ L. R. Penner 1939 chez Melospiza lincolni (Audubon); T. gallica R.-Ph. Dollfus 1946 chez Pica pica (L.), Garrulus glanda- 


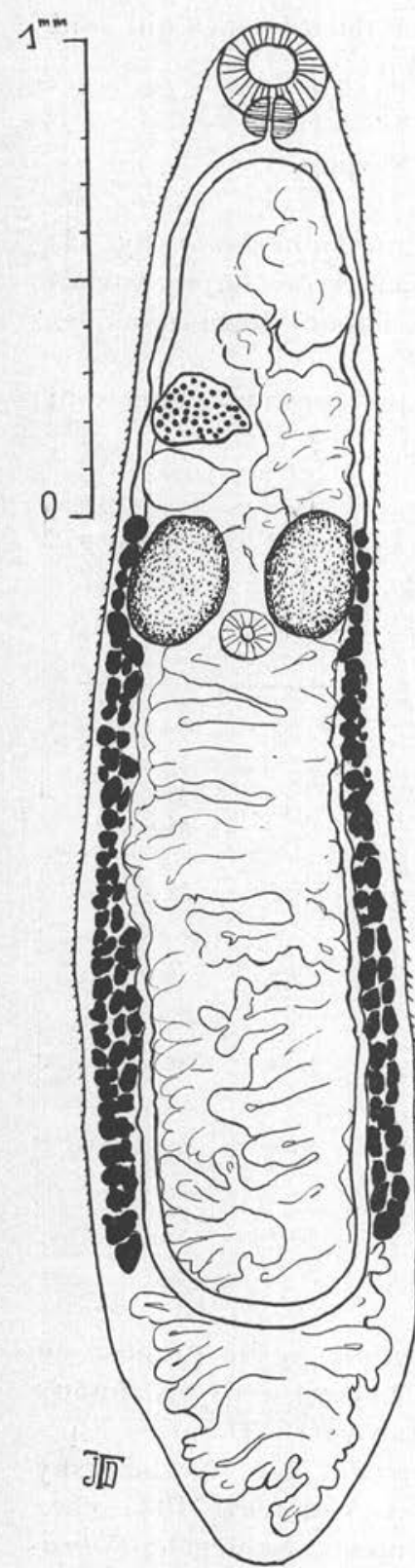

Fic. 14. - Tamerlania Za rudnyi K. I. Skrjabin. Reins et uretères de Pie. rius (L.) et Corvus corone L. Une monographie complète des Eucotylidær avec révision du genre Tamerlania a été publiée par J. F. Teixeira de Freitas (1951).

Dans sa revue de 1946 , R.-Ph. Dollfus reconnaît six genres parmi les Eucotylidæ : Eucotyle Skrjab., Tamerlania Skrjab., Tanaisia Skrjab., Ohridia Nezlobinsky, Lepidopteria Nezlob. et Nephrobius Poche. J.F. Teixeira de Freitas, se basant sur la grande variabilité des caractères relatifs fournis par la position et la forme des gonades, n'admet plus que deux genres dans la famille des Eucotylidæ: Eucotyle Cohn. et Tanaisia Skrjab. (ce dernier considéré comme synonyme de Tamerlania, Odhneria, Lepidopteria et Proshystera Korkhaus 1930). Je me bornerai à adopter ici les genres admis par R.-Ph. Dollfus en 1946.

C'est à R.-Ph. Dollfus que l'on doit la première découverte de Tamerlania chez la Pie en France, à Richelieu (Indre-et-Loire) : cinq et dix sujets chez troies Pies ; la même espèce chez le Geai et, en abondance, chez Corvus corone L.

Les exemplaires de Richelieu ont été décrits sous le nom de $T$. gallica Dollfus : la nouvelle espèce était basée essentiellement sur la présence d'un acétabulum. Par la suite, il a été reconnu qu'un acétabulum existe aussi chez $T$. zarudnyi Skrjab., comme l'a décrit et figuré J. K. Strom (1935). Il en résulte que $T$. gallica tombe en synonymie. Cette manière de voir, suggérée par R.-Ph. Dollfus lui-même (1946, p. 72), a été adoptée par J. F. Tẹixeira de Freitas (1951, p. 63).

Je rapporte donc à $T$. zarudnyi K. I. Skrjabin 1924 les sujets que j'ai trouvés dans les reins et les uretères de la Pie au Tholonet; c'est la seconde fois qu'un $\mathrm{Ta}$ merlania est signalé en France ; il est vrai- 
semblable que cette grande rareté n'est qu'apparente et due seulement à l'absence de recherches. J'ajoute que j'ai retrouvé la même espèce dans l'uretère d'un Merle (Turdus merula L.), tué dans la même localité le 21 décembre 1952. Les branches intestinales étaient bien anastomosées en anse chez cet individu, qui ne saurait être rapporté à $T$. meruli Nezlobinsky. T. zarudnyi a d'ailleurs été signalé chez un grand nombre de Passériformes (voir Teixeira de Freitas, p. 60).

L'examen des 56 sujets recueillis chez la Pie m'a permis de souligner la grande variabilité de l'espèce en ce qui concerne la forme des testicules et l'extension des vitellogènes.

Chez les individus figurés par Skrjabin (1924), les testicules et l'ovaire sont régulièrement elliptiques, à bord entier ; l'exemplaire figuré par Yamaguti (1941) a les testicules sphériques ou subspheriques et l'ovaire faiblement lobé. Les figures de R.-Ph. Dollfus (1946) montrent l'ovaire et les testicules nettement lobés. Les sujets que j'ai recueillis au Tholonet montrent les deux dispositions : la plupart ont des gonades elliptiques, à bords entiers ; chez d'autres, le contour est iranchement lobé.

L'acétabuium signalé par Strom (1935) et par Dollfus (1946) est fort difficile à voir sur les sujets mùrs; il ne peut être trouvé que sur les coupes (115 à $125 \mu)$.

L'extension des vitellogènes est assez constante en avant, où elle correspond au bord antérieur des testicules; elle varie davantage en arrière : un peu avant ou largement avant la boucle intestinale.

Les mesures que j'ai relevées sur cinq sujets sont les suivantes :

\begin{tabular}{|c|c|c|c|c|c|c|}
\hline 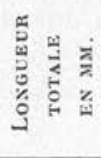 & 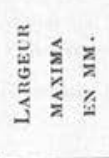 & 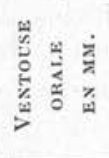 & 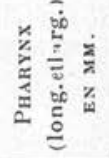 & 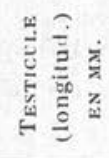 & 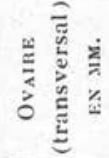 & $\sum_{0}^{n}$ \\
\hline 3,2 & 0,7 & 0,220 & $\begin{array}{l}0,084 \\
0,112\end{array}$ & 0,250 & 0,200 & $\begin{array}{c}36 \text { à } 38 \mu \\
\times 24 \mu\end{array}$ \\
\hline 2,45 & 0,56 & 0,252 & $\begin{array}{l}0,090 \\
0,112\end{array}$ & 0,182 & 0,210 & $36 \times 24 \mu$ \\
\hline 2,95 & 0,7 & 0,280 & $\begin{array}{l}0,090 \\
0,112\end{array}$ & 0,210 & 0,210 & $36 \times 24 \mu$ \\
\hline 2,66 & 0,616 & $0,2: 2$ & $\begin{array}{l}0,095 \\
0.105\end{array}$ & 0,294 & 0,210 & $36 \times 25 \mu$ \\
\hline 3,08 & 0,700 & 0,252 & $\begin{array}{l}0,098 \\
0,112\end{array}$ & 0,280 & 0,215 & $36 \times 24 \mu$ \\
\hline
\end{tabular}


Le cycle évolutif de Tamerlania bragai Dos Santos, hôte des reins et des uretères du pigeon domestique, a été découvert et reproduit expérimentalement à Porto-Rico (J.F. Maldonado, 1924 et 1925). L'hôte intermédiaire est un Gastéropode terrestre : Subulina octona (Chemnitz).

Tamerlania zarudnyi est le plus souvent localisé dans les tubes collecteurs du rein ; je l'ai aussi observé dans l'uretère (fig. 15). La présence du parasite provoque l'effacement des plis de la muqueuse et de la sous-muqueuse ; les cellules de la tunique épithéliale deviennent plus basses et présentent des lésions dues à la compression mécanique et, sans doute aussi, à l'action des toxines libérées.
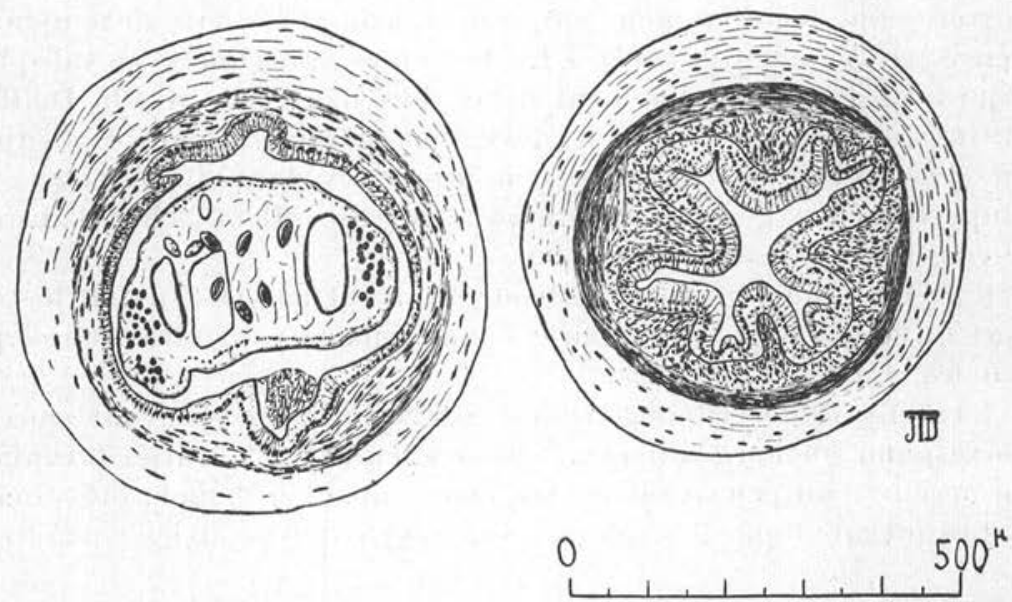

Fig. 15. - Coupe transversale d'un uretère de Pie avec Tamerlania Zarudnyi Skrjabin. Noter les lésions de la muqueuse et l'effacement des plis.

FIG. 16. - Uretère de Pie normal pour comparaison. Coupe transversale.

\section{Reaicola bretensis Timon-David 1953}

Ce Renicola (fig. 17) a été trouvé à deux reprises dans les reins de la Pie au Tholonet: une $q$ tuée le 26 septembre 1952 et un ơ le 30 septembre 1952. Le parasitisme était intense (plusieurs centaines de sujets). Les vers étaient réunis par couples dans des kystes résultant de la dilatation des tubes collecteurs. L'extraction des parasites entiers est une opération délicate, qui doit être réalisée au moyen de deux fines aiguilles sous le contrôle du binoculaire ; il faut dilacérer la paroi des kystes pour extraire leur contenu. Dans ces conditions, il est possible d'obtenir, à côté d'un grand nombre 


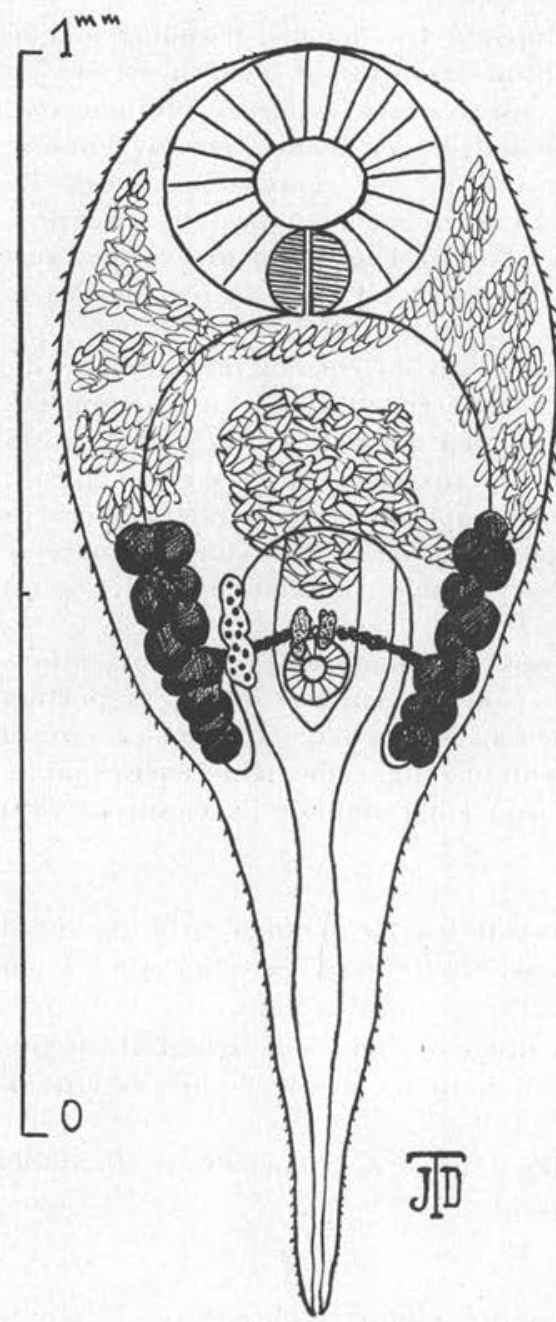

Fig. 17. - Renicolx bretensis Timon-David 1953. Reins de Pie

de mutilés, une série d'individus en bon état permettant d'en faire l'étude.

Le corps est piriforme, arrondi en avant, longuement atténué en arrière où il constitue un long prolongement caudal. Les téguments sont spinulés. Longueur moyenne : 1,2 mm. (chiffre pouvant varier notablement suivant l'extension du prolongement caudal). Largeur maxima : $0,460 \mathrm{~mm}$. au niveau de l'extrémité postérieure du pharynx. Ventouse orale un peu elliptique : son grand axe transversal peut varier de 0,252 à 0,308 mm.; en moyenne, 0,294 $\mathrm{mm}$. Le petit axe, longitudinal, est compris entre 0,210 et 0,270 mm. ; en moyenne, 0,266. L'acetabulum est faiblement excavé, visible seulement sur les coupes en série; son diamètre m'a paru très constant : $0,055 \mathrm{~mm}$. Le rapport du grand axe de la ventouse orale au diamètre de l'acetabulum est donc égal en moyenne à 5,3. Ce chiffre est très supérieur à celui que donnent toutes les espèces de Renicola décrites jusqu'ici. La distance du centre de la ventouse orale au centre de l'acetabulum est en moyenne de $0,56 \mathrm{~mm}$.

Le pore génital s'ouvre audevant de l'acetabulum; l'orifice excréteur à l'extrémité du prolongement caudal.

Le pharynx robuste est à peu près aussi long que large $(0,070 \times$ $0,070 \mathrm{~mm}$.) ; il fait suite immédiatement à la bouche : sur les préparations totales, il se montre recouvert par la ventouse orale. Il n'y a pas d'œsophage, au sens ordinaire du mot, mais une large poche qui résulte de la fusion des deux crea. Ces derniers ne s'individualisent qu'un peu plus loin; ils dépassent nettement l'acetabulum (environ $0,08 \mathrm{~mm}$. au delà de son bord postérieur). 
La vessie est en Y : la branche impaire, très longue, parcourt tout le prolongement caudal. Le point de bifurcation est un peu en arrière de l'acetabulum. Les deux branches, après s'être séparées obliquement, deviennent presque parallèles et se dirigent en avant vers la ventouse orale; leur situation est dorsale et interne par rapport aux cæca. Le nombre et la disposition des canaux excréteurs n'ont pas été observés.

Les testicules sont très rapprochés l'un de l'autre, au niveau de l'acetabulum; ils sont de petite taille; leur bord est lobé. Je n'ai pas vu la vésicule séminale.

L'ovaire est situé ventralement au-devant du cæcum intestinal droit ; il affecte la forme d'un cordon irrégulièrement lobé. Les vitellogènes sont formés de follicules assez gros et peu nombreux; ils sont disposés latéralement et dorsalement par rapport aux cæca qu'ils accompagnent ; leur extension est réduite : ils débutent après le premier tiers du corps et ne pénètrent pas dans le tiers postérieur. Le vitelloducte transverse passe immédiatement en avant de l'acetabulum. La glande de Mehlis est à côté du pore génital.

L'utérus se développe en décrivant de nombreuses circonvolutions latérales jusqu'à la hauteur du milieu de la ventouse orale ; sa portion terminale constitue un sac utérin médian bourré d'œufs, un peu en avant de l'acetabulum. Les œufs présentent une ligne de déhiscence dans le voisinage d'un de leurs pôles; ils sont embryonnés; ils mesurent 28 à $30 \mu$ sur 11,5 à $15 \mu$.

Renicola bretensis se range parmi les Renicola à prolongement caudal très iong. L'espèce est essentiellement caractérisée - en dehors de sa localisation chez la Pie - par sa ventouse orale très grande, robuste et puissamment musclée, par son acétabulum faible, par la large poche d'où se détachent les cæca et par ses vitellogènes courts.

La liste qui suit indique la répartition des espèces de Renicola actuellement connues chez les oiseaux :

\section{PASSÉriformes :}

Hirundo rustica L. : Renicola magnicaudata Bychovskaya-Pavlovskaya 1950, Sibérie.

Pica pica (L.) : R. bretensis Timon-David 1953, Le Tholonet (Bouches-du-Rhône).

Falconiformes :

Pandion halioetus L. : R. pandioni Sudarikov in Skrjabin 1947, U.R.S.S.

R. undecima Sudarikov, ibid., U.R.S.S.

Procellarifformes :

Puffinus kühlii (Boie) : R. glandoloba Witenberg 1929, Sinaï. 


\section{PODICIPITIFORMES :}

Podiceps cristatus (L.) : R. pinguis Mehlis in Creplin 1846, Allemagne.

PÉlÉCANIFormes :

Pelecanus onocrotalus L. : R. secunda Skrjabin 1924, Turkestan.

ARdérformes :

Bubulcus coromandus (Boddaert) : Renicola sp. Hammerton 1934, Londres.

ANSÉRIFORMES :

Nyroca ferina (L.) : $R$. mediovitellata Bychovskaya-Pavlovskaya 1950, Sibérie.

Spatula clypeata (L.) : R. mediovitellata Bych.-Pavl. 1950, Sibérie. Anas strepera L. : R. mediovitellata Bych.-Pavl. 1950, Sibérie.

LARIFORMES :

Larus argentatus michaellis Naumann : R. lari Timon-David 1933, Marseille, île de Riou (très commun).

Larus ridibundus L. : R. paraquinta Rayevsky 1937, Tobolsk (Oural). Sterna hirundo L. : R. tertia Skrjabin 1924, Turkestan.

Sterna cantiaca Gm. : Renicola sp. Callot 1946, Côtes de la Manche.

\section{Alciformes :}

Mergulus alle (L.) : Renicola sp. Dollfus 1912, Wimereux (Pas-deCalais).

Uria aalge inornata Salom. : R. umigarasu Yamaguti 1939, Japon.

Cepphus carbo Pallas : R. keimahuri Yamaguti 1939, Japon.

R. quinta Sokolowa-Andronowa 1937, Vladivostok.

Cyclorrhynchus psittacula Pallas: Renicola sp. Sokolowa-Andronowa 1937 , Vladivostok.

\section{LÉsions RÉNALES PROVOQUÉEs CHEZ LA PIE PAR Renicola bretensis T.-D.}

On sait que le rein des oiseaux est constitué par la juxtaposition de lobules distincts ; dans chacun d'eux, les tubes médullaires sont groupés par faisceaux et coiffés par une épaisse couche corticale à très nombreux glomérules. C'est aux dépens des canalicules afférents, au point où ils rejoignent les tubes collecteurs, que prennent 
naissance les kystes à Renicola bretensis; ils provoquent en cet endroit une énorme dilatation de la paroi qui aboutit à la formation de diverticules ovoïdes. Ces kystes se développent en refoulant le cortex, dans lequel ils pénètrent assez profondément. Il est possible de dissocier, sous le contrôle du binoculaire, un tube collecteur avec les kystes qui y sont annexés (fig. 18). L'aspect est alors celui d'une sorte de grappe très caractéristique, les kystes étant suspendus aux canalicules afférents. Chacun d'eux renferme toujours une paire de

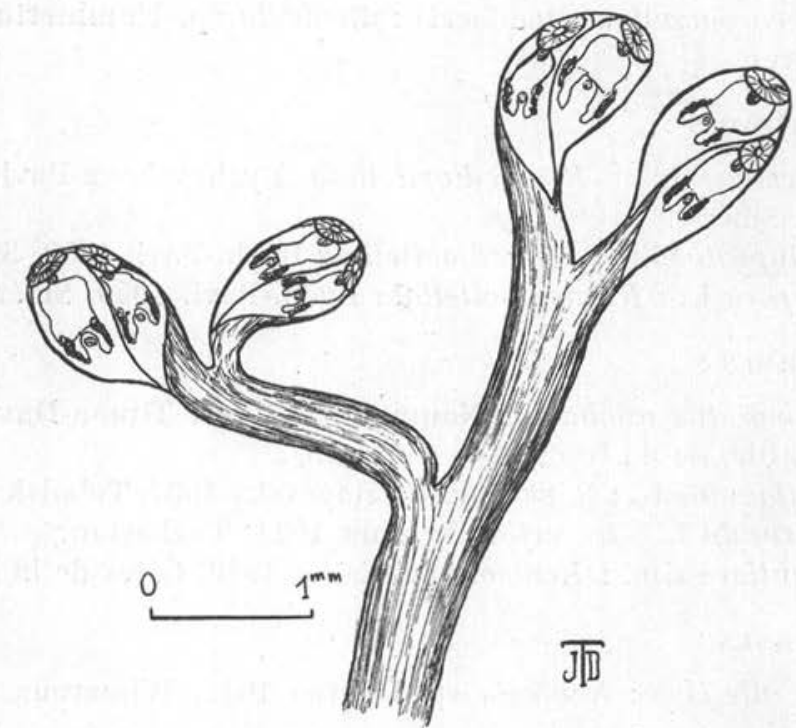

Fıg. 18. - Kystes à Renicola bretensis T.-D., isolés par microdissection au binoculaire. Disposition « en grappe » caractéristique.

parasites. Dans le voisinage, le tissu glomérulaire présente des lésions de tassement et de compression qui peuvent intéresser plusieurs lobules voisins.

La paroi kystique est constituée par l'épithélium tubulaire plus ou moins modifié et altéré : les cellules plus basses, à noyaux subcentraux, diffèrent sensiblement des éléments normaux qui sont cylindriques avec noyaux basaux.

Tant que les kystes renferment des parasites vivants, leur paroi ne présente pas de réaction leucocytaire; celle-ci devient intense dans les nodules qui se développent autour des Renicola morts et dégénérés. Il se forme dans ce cas un revêtement scléreux avec infiltration leucocytaire abondante. Dans ces kystes en dégénérescence, les œufs persistent sans altération, tandis que les autres 

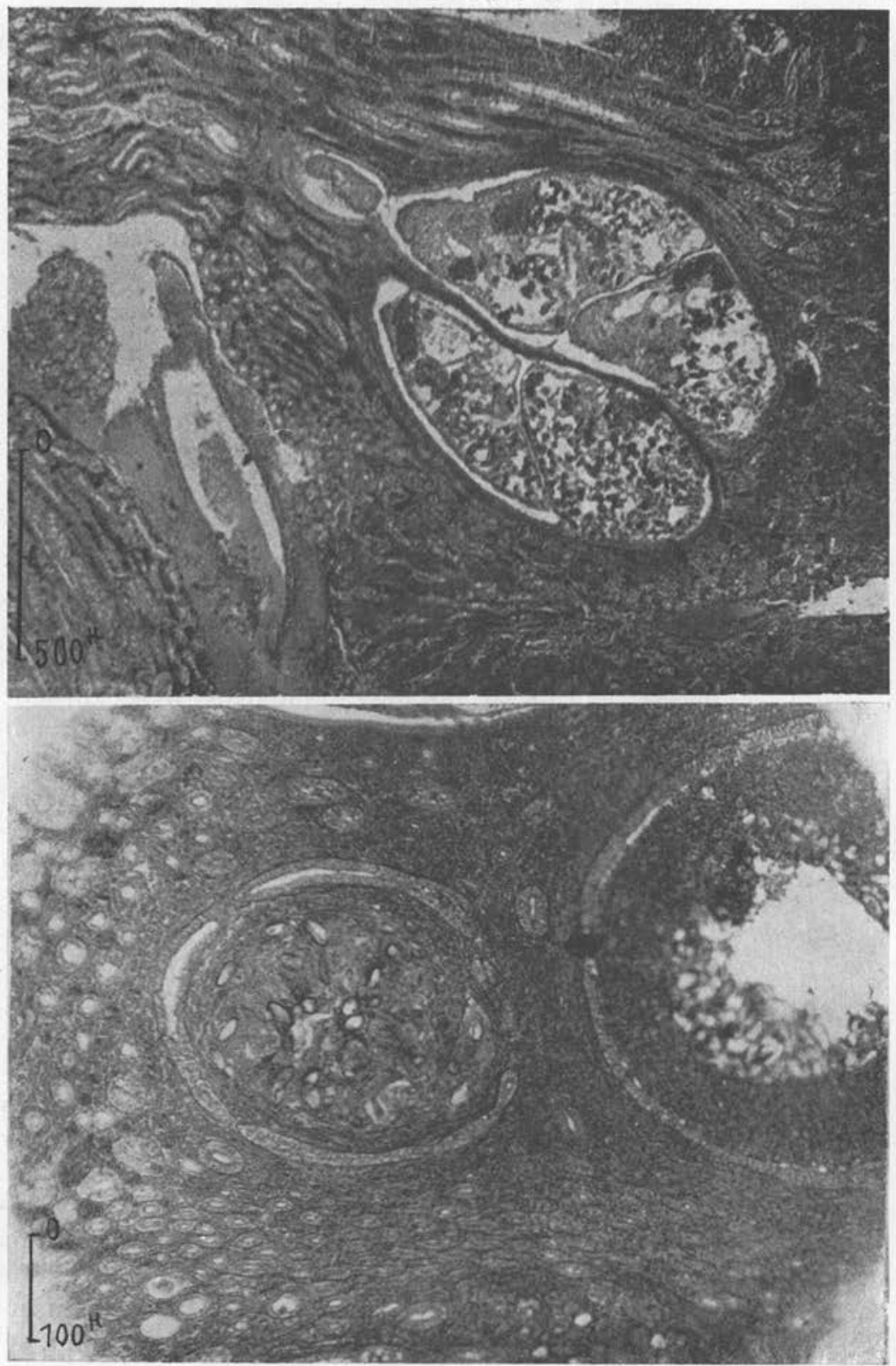

Fig. 19 (en haut). - Rein de Pie avec kystes à Renicola brelensis T.-D.

F1G. 20. - Vieux nodule kystique à Renicola bretensis T.-D. Les tissus du parasite sont désorganisés: sẹls les œufs persistent en bon état. 
organes du parasite subissent une lyse de plus en plus accusée (fig. 20). Les œufs peuvent, dans ce cas, se trouver disséminés dans

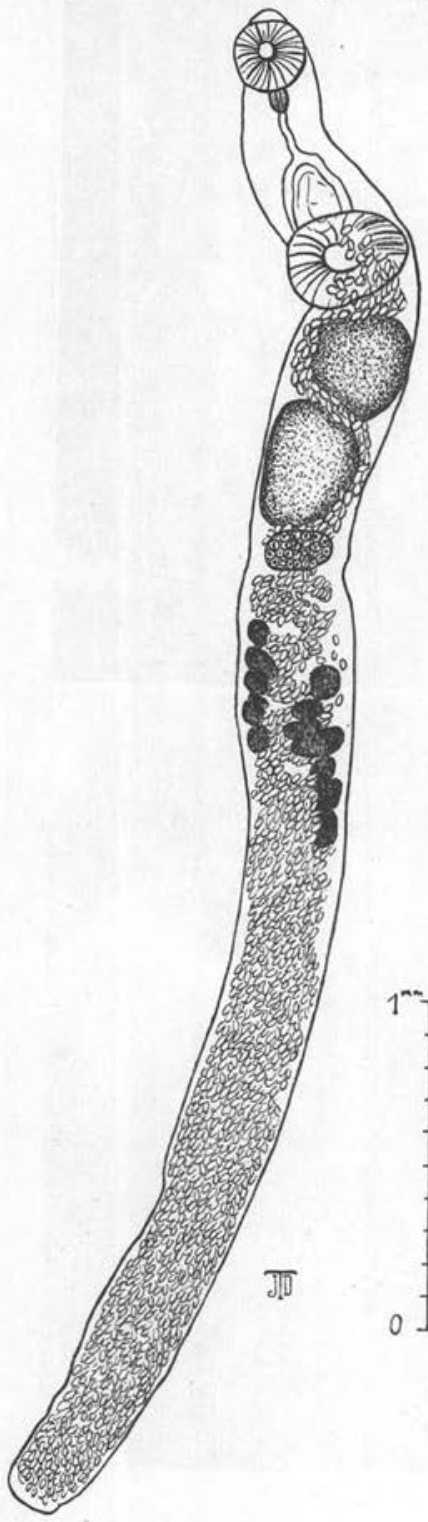

FIG. 21. - Brachylecithum lobatum (Railliet). Canalicules biliaires intra-hépatiques de Pie. le tissu glomérulaire.

\section{V. - Trématodes du foie et des voies biliaires}

\section{Brachylecithum lobatum \\ (Railliet 1900) \\ (= Olssoniella lobata (Raill.) in Travassos 1944)}

J'ai trouvé très fréquemment ce Dicrocœlien dans le foie de la $\mathrm{Pie}$ : chez 61 p. 100 des oiseaux examinés, parfois en grande abondance. Les parasites étaient logés dans les canalicules biliaires, d'où il est assez difficile de les extraire sans les déchirer, par suite de leur longueur et de leur fragilité. J'ai pu préparer un matériel important: une centaine d'exemplaires entiers; mais il va sans dire que d'autres, bien plus nombreux, ont été mutilés ou perdus. J'ai également prélevé des pièces histologiques pour étudier les lésions hépatiques.

La littérature concernant cette espèce est assez compliquée (voir Travassos, 1944, p. 209-210). Il y a eu diverses confusions, en particulier avec Lyperosomum longicauda (Rud.). L'amplitude des variations anatomiques est mal connue et je me suis efforcé de la préciser d'après une série d'exemplaires convenablement conservés. Le cycle évolutif est inconnu.

Dicrocoelium lobatum Railliet a été longtemps attribué au genre Lyperosomum Looss 1899 sensu lato; ce genre a été démembré par Travas- 


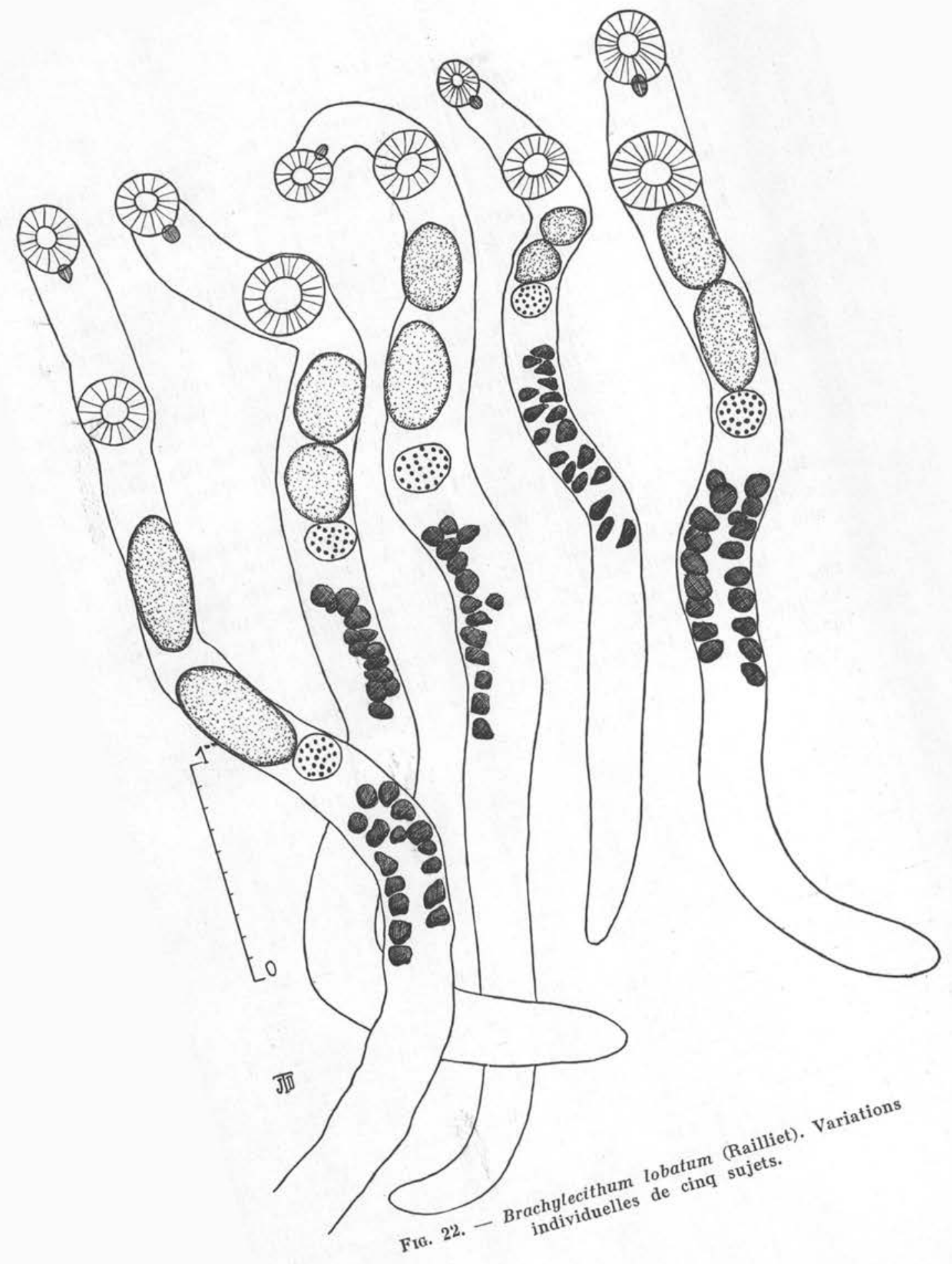


sos (1944) en Lyperosomum sensu stricto, Zonorchis Travassos, Orthorchis Travassos, Olssoniella Trav., et Lutztrema Trav. L'auteur brésilien attribue $D$. lobatum à son genre Olssoniella, caractérisé par la position du pore génital en arrière de la bifurcation intestinale ou à son niveau, par la brièveté de l'œsophage, par les vitellogènes en gros follicules postovariens, par les testicules situés l'un au-devant de l'autre sur une même ligne droite, par les sinuosités prétesticulaires de l'utérus.

Dollfus (1951, p. 148-149) a fait remarquer que d'autres noms de genres, proposés par J. K. Strom (1940), dans un travail dont Travassos n'a pu avoir connaissance, ont la priorité : Olssoniella doit être remplacé par Brachylecithum Strom, et Orthorchis par Corrigia Strom 1940. C'est donc la dénomination de Brachylecithum lobatum (Railliet) que j'adopterai ici.

Travassos (1944, p. 210) donne comme localisation pour O. lobata uniquement la vésicule biliaire. Je n'ai jamais trouvé ce Distome dans la vésicule, mais toujours dans les canalicules intra-hépatiques. Railliet (1900) indique bien le foie, et Braun (1902, p. 110) dit aussi « die Leber ». Samenow (1927) mentionne également le foie pour son Lyperosomum lobatum glandarii.

Les hôtes cités sont : Pica pica (L.), Garrulus glandarius (L.), Corvus cornix (L.), Corvus corone (L.), Coloeus monedula (Kaup), et un Accipitriforme : Accipiter nisus (L.). Le type décrit par Railliet provenait de ce dernier hôte.

Voici les mensurations que j’ai relevées sur cinq sujets :

\begin{tabular}{|c|c|c|c|c|c|c|c|}
\hline 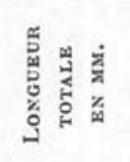 & 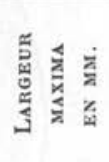 & 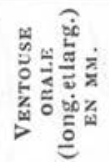 & 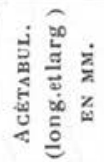 & 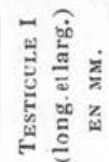 & 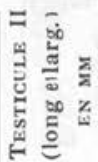 & 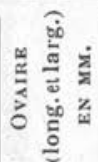 & 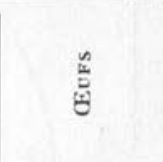 \\
\hline 425 & 0,308 & $\begin{array}{l}0,226 \\
0,168\end{array}$ & $\begin{array}{r}0,224 \\
0,350\end{array}$ & $\begin{array}{l}0,350 \\
0,210\end{array}$ & $\begin{array}{l}0,322 \\
0,252\end{array}$ & $\begin{array}{l}0.168 \\
0.210\end{array}$ & $\begin{array}{c}45,6 \mu \times 28,8 \mu \\
45,6 \times 31,2 \mu\end{array}$ \\
\hline 420 & 0,210 & $\begin{array}{l}0,224 \\
0,196\end{array}$ & $\begin{array}{l}0,224 \\
0,252\end{array}$ & $\begin{array}{l}0,224 \\
0,112\end{array}$ & $\begin{array}{l}0,252 \\
0140\end{array}$ & $\begin{array}{l}0,168 \\
0,112\end{array}$ & $\begin{array}{l}43,2 \times 26,4 \mu \\
45,6 \times 27,6 \mu\end{array}$ \\
\hline $\begin{array}{c}\text { Sujet } \\
\text { incomplet }\end{array}$ & 0,310 & $\begin{array}{l}0,294 \\
0,252\end{array}$ & $\begin{array}{l}0,350 \\
0,350\end{array}$ & $\begin{array}{l}0,588 \\
0,261\end{array}$ & $\begin{array}{l}0,580 \\
0,261\end{array}$ & $\begin{array}{l}0,252 \\
0.252\end{array}$ & $\begin{array}{l}43,2 \times 26,4 \mu \\
48 \times 26,4 \mu\end{array}$ \\
\hline id. & 0,305 & $\begin{array}{l}0,200 \\
0,200\end{array}$ & $\begin{array}{r}0,260 \\
0,380\end{array}$ & $\begin{array}{l}0,350 \\
0,300\end{array}$ & $\begin{array}{l}0.350 \\
9,300\end{array}$ & $\begin{array}{l}0,120 \\
0,200\end{array}$ & $\begin{array}{c}43,2 \times 26,4 \mu \\
48 \times 28,2 \mu\end{array}$ \\
\hline id. & 0,245 & $\begin{array}{l}0,210 \\
0,196\end{array}$ & $\begin{array}{l}0,266 \\
0,252\end{array}$ & $\begin{array}{l}0,196 \\
0,196\end{array}$ & $\begin{array}{l}0,196 \\
0,196\end{array}$ & $\begin{array}{l}0,168 \\
0,168\end{array}$ & $\begin{array}{l}43,2 \times 28,8 \mu \\
45,6 \times 28,8 \mu\end{array}$ \\
\hline
\end{tabular}




\section{LES LÉSIONS HÉPATIQUES PROVOQUÉES PAR}

\section{Brachylecithum lobatum (Railliet)}

Aucun auteur n'a observé jusqu'ici la localisation de $B$. lobatum (Railliet) dans le foie de la Pie et n'a décrit les lésions provoquées.

Mes coupes (fig. 23) montrent que les parasites ne sont jamais isolés dans les canalicules biliaires : ils sont toujours groupés par paquets de trois à six, étroitement serrés et accolés les uns aux autres. Le canalicule subit, naturellement, une dilatation considérable : son diamètre peut atteindre $520 \mu$.

A l'endroit où sont logés les parasites, la paroi épithéliale peut être complètement détruite ; la cuticule du ver est alors en contact direct avec la tunique conjonctive fortement épaissie et sclérosée (19 à $20 \mu$ sur les coupes colorées à la picro-fuchsine). Ailleurs, dans le voisinage de la ventouse orale, l'épithélium biliaire peut être conservé, mais il est profondément altéré : les noyaux déformés, elliptiques ou excavés en cupule, comprimés ou écrasés.

Cette hypertrophie détermine aussi des lésions de tassement et d'écrasement dans le voisinage; les autres canalicules biliaires de l'espace porte n'ont plus qu'une lumière étroitement allongée, qui peut même disparaître entièrement, les deux côtés du revêtement épithélial du tube arrivant au contact. Tout autour, les lobules hépatiques sont aussi écrasés et déformés. La leucocytose est très accusée ; les veines interlobulaires sont bourrées de leucocytes.

\section{Cycle évolutif}

Le cycle de Brachylecithum lobatum est inconnu, mais il est intéressant de signaler que celui d'une espèce voisine, $B$. americanum Denton, a été élucidé en 1945 par J.-F. Denton. Ces recherches peuvent aider à découvrir le cycle de notre espèce.

B. americanum vit aux Etats-Unis (Géorgie, Texas, Tennessee) dans les canalicules biliaires du foie de certains Corvidés et Ictéridés. Les œufs se développent expérimentalement chez des Gastéropodes terrestres : Polygyra texasiana (Moricand) et Practicollela berlanderiana (Moricand). Deux générations de sporocystes se succèdent chez ces Mollusques; les derniers émigrent dans la paroi de la cavité palléale ; les cercaires mûres se rassemblent dans la cavité elle-même et sont finalement expulsées par le pneumostome sous forme d'amas de 150 à 300 , qui sont déposés sur diverses plantes. Le nombre des cercaires ainsi éliminées est considérable : il a été évalué à plus de 20.000 pour un seul Polygyra texasiana. 

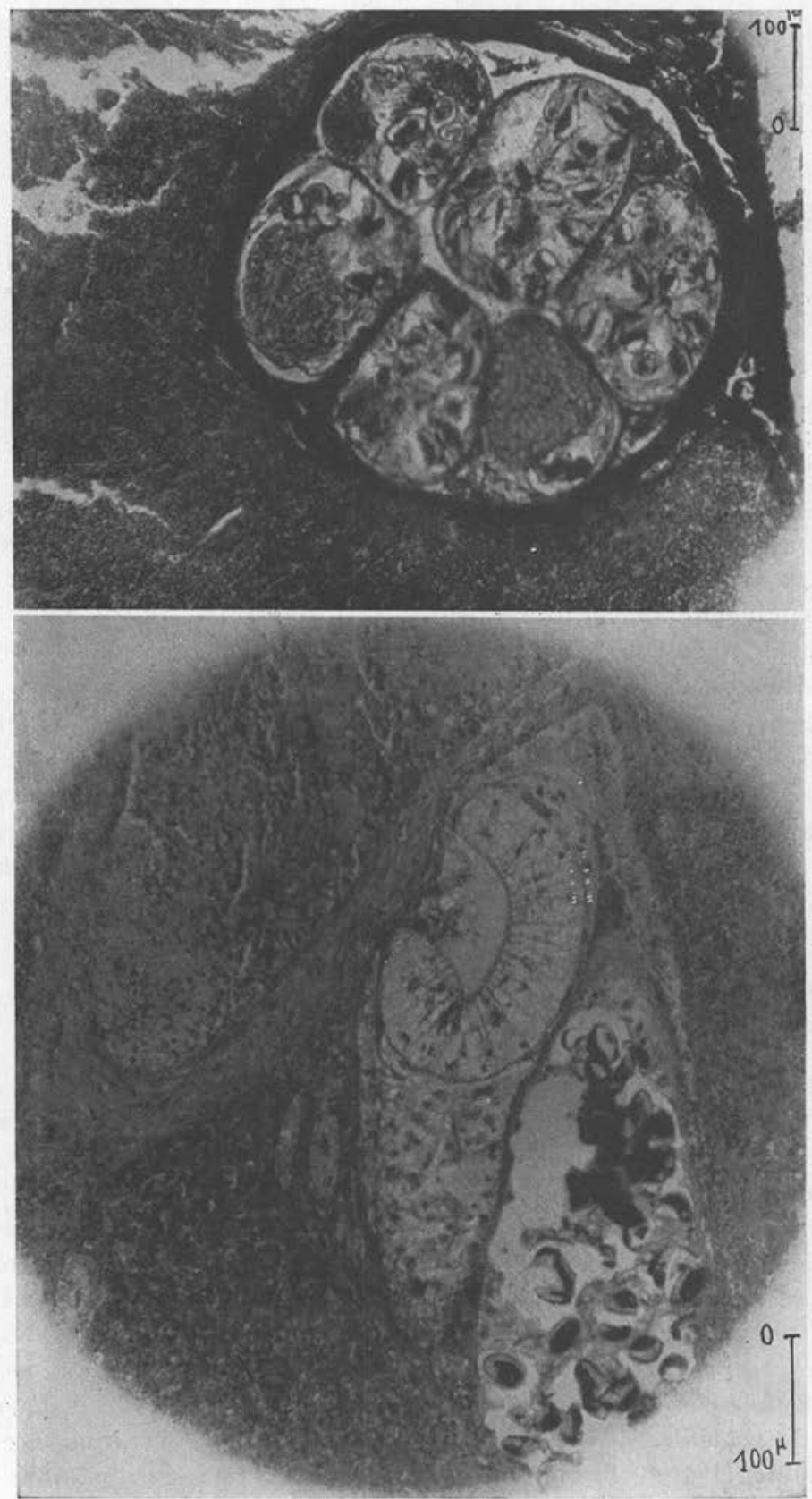

Fig. 23 (en haut), - Foie de Pie parasité par Brachylecithum lobatum (Railliet). La coupe montre un canalicule biliaire hypertrophié dans lequel sont juxtaposés six sujets étroitement serrés.

FIG. 24. - Foie de Pie avec Brachylecithum lobatum (Railliet). 
Le second hôte est représenté par des larves de Chrysomélides : Diabrotica duodecimpunctata et Gastroidia cyanea. Il est extrêmement probable que les Corvidés se contaminent en mangeant ces Coléoptères.

\section{Lyperosomum petiolatum (Railliet 1900)}

Trouvé une seule fois chez la Pie : huit exemplaires dans la vésicule biliaire.

Le type de Railliet avait été recueilli chez le Geai en Seine-etMarne; l'espèce a été retrouvée chez le même hôte à Richelieu (Indre-et-Loire) par R.-Ph. Dollfus, Callot et Desportes (1934, p. 524) ; elle a été citée également chez le Merle (Travassos, 1934, p. 137), mais ne paraît pas avoir été signalée chez la Pie.

Mes échantillons concordent bien avec les descriptions de Railliet et de Braun (1902, p. 98). Les chiffres relevés sont les suivants :

\begin{tabular}{|c|c|c|c|c|c|c|c|c|c|}
\hline 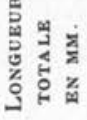 & 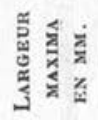 & 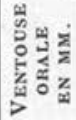 & 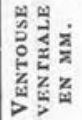 & 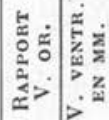 & 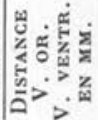 & 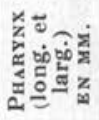 & 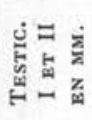 & 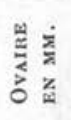 & 离 \\
\hline 7,0 & 1,38 & 0,380 & 0,833 & $1: 2,19$ & 1,38 & $\begin{array}{l}0,140 \\
0,168\end{array}$ & $\begin{array}{l}0,380 \\
0,428\end{array}$ & 0,380 & $\begin{array}{l}48 \mu \mathrm{a} \\
50 \mu \mathrm{a} \\
25 \mathrm{a} \\
26 \mu\end{array}$ \\
\hline 7,07 & 1,23 & 0,378 & 0,644 & $1: 1,7$ & 1,33 & $\begin{array}{l}0,168 \\
0,168\end{array}$ & $\begin{array}{l}0,320 \\
0,580\end{array}$ & 0,380 & $\begin{array}{l}45,6 \mu \times \\
28,8 \mu\end{array}$ \\
\hline 6,7 & 1,26 & 0,385 & 0,784 & 1: 2,0 & 1,26 & $\begin{array}{l}0.140 \\
0,168\end{array}$ & $\begin{array}{l}0,365 \\
0,450\end{array}$ & 0,350 & $\begin{array}{l}43, \mu \times \\
28,8 \mu\end{array}$ \\
\hline
\end{tabular}

C'est peut-être à Lyperosomum petiolatum (Railliet) que correspondent les cercaires et métacercaires du type vitrina Linst., observées chez Helix aspersa O. F. Müller à Richelieu par Dollfus, Callot et Desportes (1934).

\section{Lyperosomum longicauda (Rudolphi 1809)}

Je réunis sous ce nom une série de Dicrocœliidés recueillis au Tholonet dans la vésicule biliaire de trois Pies (jusqu'à sept sujets chez le même hôte).

La description originale de Rudolphi a été reprise par une série d'auteurs, en particulier Mühling (1896, p. 248), Railliet (1900, 


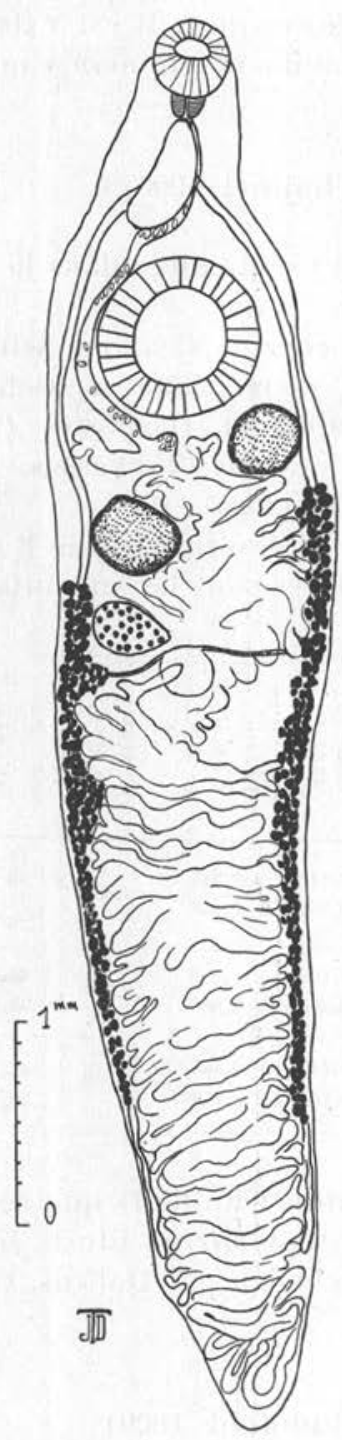

Fig. 25. - Lyperosomum petiolatum (Railliet). Vésicule biliaire de Pie.

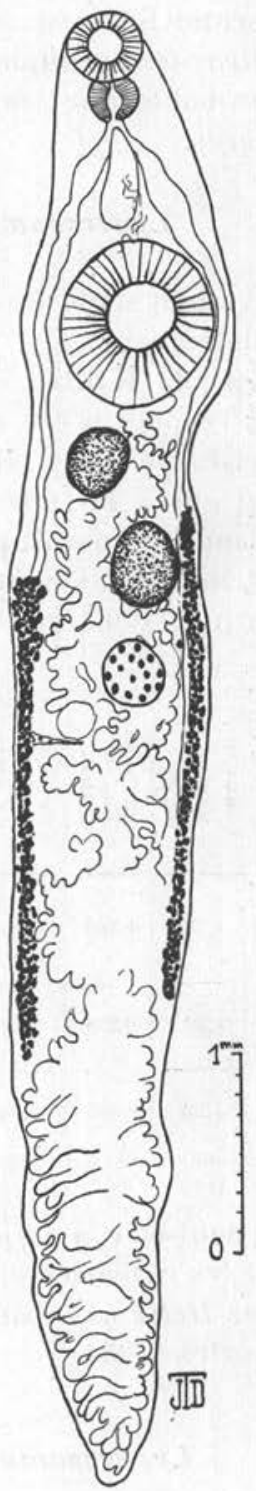

Fig. 26. - Lyperosomum longicauda (Rud.). Vésicule biliaire de Pie.

p. 240), Braun (1902, p. 106-109), Travassos (1944, p. 132). On trouvera dans ce dernier travail la synonymie complète. 
Mes exemplaires (fig. 26) sont un peu plus petits que le type de Rudolphi ; ils paraissent identiques à la forme signalée par Railliet chez Corvus cornix (L.) et chez Trypanocorax frugilegus (L.). Le type de Rudolphi avait été trouvé dans la vésicule biliaire de Corvus cornix (L.) ; l'espèce est citée chez Corvus corone (L.) et chez Pica pica (L.) (W. Nicoll, 1923, p. 172-173).

Le tableau ci-dessous donne les mensurations de trois exemplaires :

\begin{tabular}{|c|c|c|c|c|c|c|c|c|c|}
\hline 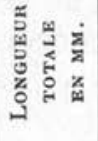 & 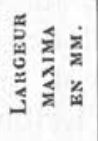 & 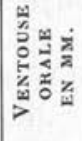 & 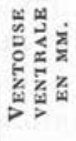 & 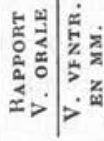 & 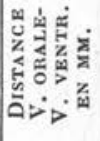 & 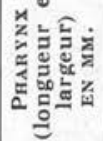 & 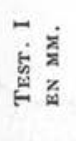 & 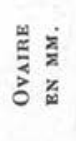 & $\stackrel{\infty}{\sum_{\theta}^{\infty}}$ \\
\hline 7,3 & 1,05 & 0,378 & 0,756 & $1: 2$ & 1,372 & $\begin{array}{l}0,210 \\
0,224\end{array}$ & 0,240 & 0,310 & $\begin{array}{l}31,2 \mu \times \\
21,6 \mu\end{array}$ \\
\hline 5,95 & 0,728 & 0,280 & 11,560 & $1: 2$ & 1,008 & $\begin{array}{l}0,160 \\
0,210\end{array}$ & 0,280 & 0,252 & $\begin{array}{l}36 \mu \times \\
24 \mu\end{array}$ \\
\hline 5,67 & 0,938 & 0,406 & 0,826 & $1: 2$ & 1,232 & $\begin{array}{l}0,210 \\
0,252\end{array}$ & 0,365 & 0,280 & $\begin{array}{c}36 \mu \times \\
24 \mu\end{array}$ \\
\hline
\end{tabular}

\section{Zonorchis panduriforme Railliet 1900}

Je crois pouvoir rapporter à cette espèce une dizaine de Distomes trouvés dans la vésicule biliaire de trois Pies.

Dicrocœlium panduriforme a été sommairement décrit par Railliet (1900) d'après deux sujets recueillis dans la vésicule biliaire d'une Pie tuée dans l'Aisne. Malheureusement, cette description n'a été accompagnée d'aucune figure. L'espèce ne semble pas avoir été revue depuis. Dans sa monographie de 1944 , L. Travassos (p. 41) se borne à recopier et à interpréter la description de Railliet, qui est la suivante : "Corps en forme de violon, offrant un étranglement très prononcé au niveau du tiers postérieur et prolongé en avant par un cou cylindro-conique, se recourbant volontiers en dessous et en arrière. L'extrémité postérieure est très obtuse. Ventouse buccale subterminale, infère, large de $320 \mu$. Ventouse ventrale très saillante, large de $500 \mu$, à ouverture circulaire. Testicules situés presque côte à côte, en arrière de la ventouse ventrale. Ovaire aussi volumineux que les testicules, situé à peu de distance en arrière du testicule droit. Vitellogènes en bandes continues, d'abord assez larges, puis s'atténuant pour se terminer vers le milieu du renflement 


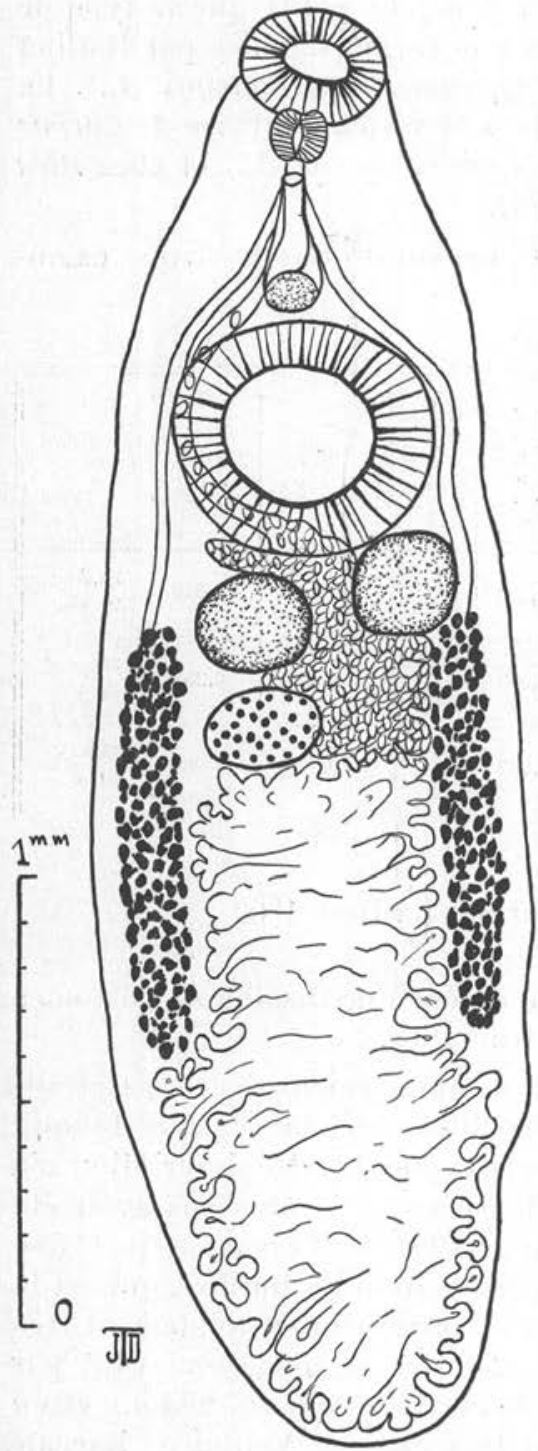

FIG. 27. - Zonorchis panduriforme (Railliet). Vésicule biliaire de Pie. postérieur. Pore génital en arrière du pharynx. Cirre long de $140 \mu$, large de $70 \mu$. CEufs : 42 à $45 \mu \times 22$ à $25 \mu$. 》.

Il est évident, comme le suggère L. Travassos (loc. cit.), que cette forme anormale est un artefact résultant de la fixation. En dehors de cette particularité, les caractères des sujets que j'ai recueillis au Tholonet concordent sensiblement avec la description de Railliet; les dimensions sont voisines et il est permis de penser qu'il s'agit bien de la même espèce.

Dans sa définition des genres de Dicrocceliidæ, L. Travassos (p. 15) réserve le nom de Dicrocolium aux formes à ventouses égales ou subégales (rapport compris entre $1: 1$ et $1: 1,3)$ et à testicules situés obliquement. L'espèce de Railliet ne peut donc être maintenue dans ce genre, et il est difficile d'expliquer pourquoi l'auteur brésilien a conservé cette dénomination. Chez $\mathrm{Zo}$ norchis Travassos 1944 (page 153), le rapport $\frac{\text { ventouse orale }}{\text { acetabulum }}$ est supérieur à $1: 1,4$, et les testicules sont alignés côte à côte dans une même zone postacétabulaire. Ces caractères se retrouvent chez $D$. panduriforme. 


\begin{tabular}{|c|c|c|c|c|c|c|c|c|}
\hline 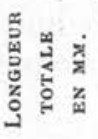 & 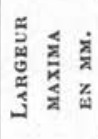 & 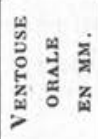 & 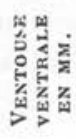 & 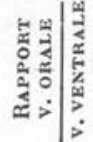 & 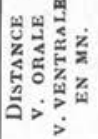 & 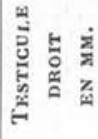 & 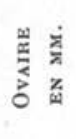 & 竞 \\
\hline 3,10 & 0,980 & 0,322 & 0,630 & $1: 1,96$ & 0,840 & 0,280 & 0,280 & $43 \mu \times 28,8$ \\
\hline 3,64 & 0,812 & 0,308 & 0,530 & $1: 1,72$ & 0,910 & 0,210 & 0,280 & $40,8 \times 26,4$ \\
\hline 4,58 & 1,134 & 0,308 & 0,630 & $1: 1,95$ & 0,910 & 0,210 & 0,280 & $43,2 \times 26,4$ \\
\hline 3,99 & 0,900 & 0,252 & 0,530 & $1: 2,1$ & 0,840 & 0,200 & 0,154 & $38,4 \times 26,4$ \\
\hline
\end{tabular}

Zonorchis panduriforme (Railliet)

Mensurations de quatre sujets.

\section{Résumé}

Des recherches sur les Helminthes de la Pie en Provence (environs du Tholonet, Bouches-du-Rhône) montrent que cet oiseau héberge une faune de Trématodes riche et variée. Onze espèces ont été trouvées, parmi lesquelles trois nouvelles; elles se répartissent de la façon suivante :

I. Trématodes des sacs aériens: Cyclocœlum (Pseudhyptiasmus) Dollfusi Timon-David 1950. Un matériel abondant a permis de préciser l'amplitude des variations individuelles et de faire une étude anatomique et histologique détaillée.

II. Trématodes du tube digestif: Brachylæmus fuscatus (Rud.). Les métacercaires de cette espèce sont hébergées par Helicopsis arenosa (Ziegler) : ce Pulmoné est fréquemment mangé par la Pie qui se contamine facilement.

III. Trématodes de la bourse de Fabricius : deux espèces. La plus commune est Prosthogonimus Brauni Skrjabin; la seconde, moins fréquente : $P$. cuneatus (Rud.). Ces observations permettent de considérer la Pie comme susceptible de jouer le rôle de réservoir de virus pour la prosthogonimose des volailles.

IV. Trématodes des reins et des uretères: Metacercaria renalis nov. sp., enkystée dans les reins, évolue peut-être chez un Falconiforme. Tamerlania zarudnyi Skrjabin est signalé ici pour la première fois dans le Sud-Est de la France; sa localisation dans les canaux urinaires et dans l'uretère détermine des lésions qui sont 
étudiées. Renicola bretensis Timon-David 1953 est la première espèce du genre signalée en France chez un Passériforme; son étude anatomique est complétée par la description des lésions qu'elle provoque dans le rein.

V. Trématodes du foie et de la vésicule biliaire : quatre espèces. Brachylecithum lobatum (Railliet) est très fréquent dans la localité (chez 61 p. 100 de Pies). Les nombreux sujets recueillis permettent de préciser les variations de l'espèce qui n'étaient pas connues. Les coupes du foie montrent les parasites in situ dans les canalicules biliaires et permettent l'étude anatomo-pathologique de l'organe. Trois autres Dicrocœliidés: Lyperosomum longicauda (Rud.), L. petiolatum (Railliet) et Zonorchis panduriforme (Railliet), sont localisés dans la vésicule biliaire. La dernière espèce est rapportée à une forme très sommairement décrite par Railliet; l'auteur en donne des mensurations et une figure.

\section{INDEX BIBLIOGRAPHIQUE}

AdaM (W.) et Leloup (E.). - Recherches sur les parasites des Mollusques terrestres de Belgique. Trématodes larvaires. Mém. Mus. Roy. Hist. Nat. Belgique, $1934, \mathrm{n}^{\circ} 62$, p. 1-40.

BaEr (J.-G.). - Contribution à la faune helminthologique de la Suisse. Rev. Suisse de Zool., 39, 1932, p. 1-56.

Вазкакоv (V. P.). - Ueber die individuelle Variabilität des Saugwurmes Prosthogonimus ovatus (Rud.). Sammlung helminthologisches Arbeiten Prof. D. K. I. Skrjabin von seiner Schülern gewidmet. Moscou, 1927, p. 25-43, fig. 1-7.

Braun (M.). - Trematoden der Bursa Fabricii, des Eileiters und Eier der Vögel. Centralbl. für Bakter, 29, 1901.

- Fascioliden der Vögel. Zool. Jahrb., Abt. f. System. Bd., 16, 1902.

Bychovskaya-Pavlovskaya (I. E.). - - New species of kidney parasites belonging to the genus Renicola, occuring in Birds. C.R. Ac. Sc. U.R.S.S. Moscon, 71,1950 , p. $415-416$ (en russe).

Brip (E. E.). - The trematode genus Tamerlania in resident birds of the United States. Journ. of Parasitol., 33, 1947, p. 16-17.

Brrd (E. E.) et Denton (J. F.). - The helminthes parasites of birds. A review of the Trematode genus Tanaisia Skrjabin 1924. Amer. Midl. Nat. NotreDame, 43, 1950, p. 32-57.

Callot (J.). - Matériaux pour servir à la faune des Distomes de France. Ann. Parasitol., XXI, 1946, p. 149.

Canavan (W. P. N.). - On a Trematode Allopgge undulatus n. sp., parasitic in Lilford's Crane (Megalornis grus Lilfordi). Parasitology, XXVI, 1934, p. 117-120.

CREPLin (F. C. H.). - Nachträge zu Gurtl's Verzeichniss der Thiere bei welchen Entozoen gefunden worden sind. Arch. für Naturgesch., XII, 1846, p. 129-160. 
Dawes (Ben), - The Trematoda. Cambridge Unịversity Press, 1946.

Denton (J. F.). - Studies on the life history of a Dicrocoeliid Trematode of the genus Lyperosomum. Journ. of Parasitol., 27, 1941, p. 13-14.

- Studies on the life history of Brachylecithum americanum n. sp. a liver fluke of Passerine Birds. Journ. of Parasitol., 31, 1945, p. 131-141.

Doli.fus (R.-Ph.). - Variations dans la forme du corps, la position et la forme des testicules chez Dicrocœlium lanceolatum (Rudolphi). Observations sur la position systématique de quelques Dicrocœliinæ. Nécessité d'une révision systématique du genre et de la sous-famille des Dicrocæliinæa (Looss) Odhner emend. Bull. Soc. Zool. Fr., XLVII, 1923, p. 312-342.

- Sur quelques Brachylamus de la faune française récoltés principalement à Richelieu (Indre-et-Loire). Ann. Parasit. Hum. et Comp., XII, 1934, p. 551-575, et XIII, p. 52-79.

Distome d'un abcès palpébro-orbitaire chez une panthère. Possibilités d'aftinités lointaines entre ce Distome et les Paragonimida. Ann. Parasitol. Hum. et Comp., XVII, 1939, p. 209-235.

- Sur un Distome du genre Tamerlania K. I. Skrjabin 1924. Avec un catalogue des Trématodes des reins d'Oiseaux. Ann. Parasitol., XXI, 1946, p. 25-73,

- Sur les Prosthogonimine, Trématodes de la Bourse de Fabricius des Oiseaux et leur biogéographie. Mém. Mus. Nat. Hist. Nat., XXIV, 1948, p. $1-73,9 \mathrm{pl}$.

- Sur deux Monostomes (Cyclocœlide) pourvus d'une ventouse ventrale. Observations sur la classification des Cyclocœloidea Albert Henry 1923. Liste des hôtes, répartition géographique. Ann. Parasitol., XXIII, 1948, p. 129-199.

- Quelques Trématodes, Cestodes et Acanthocéphales. Arch. Inst. Pasteur du Maroc, IV, 1951, p. 104-235.

Dubors (G.). - Description de deux espèces nouvelles de Cyclocœlida, suivie d'une note sur Hyptiasmus ominosus (Koss.). Rev. Suisse de Zool., XXXVII, 1930 , p. 385-395.

Fernando (W.). - Cyclocœlum (Pseudhyptiasmus) sinhaladvipa n. sp. a parasite from the nasal sinus of the Ceylon Jungle Fowl. (Gallus lafayetti). Ceylon J. Sci. (B.) Zool., 24, 1950, p. 127-129, 2 pl.

Fuhrmans (O.). - Trematoda in Kunenthal: Handbuch der Zoologie, 1928, Bd. 11 .

Harrah (E. C.). - North American Monostomes primarily from fresh water hosts. Univ. of Illinois Bull., VII, 1922, p. 231-234.

Joyeux (Ch.) et BAER (J.-G.). - Note sur les Cyclocœlida (Trématodes). Bull. Soc. Zool. Fr., LII, 1927, p. 416-434.

Joyeux (Ch.), BAER (J.-G.) et Timon-David (J.). - Recherches sur les Trématodes du genre Brachylamus (Harmostomum). Bull. Biol. Fr. et Belg., LXVIII, 1934 , p. $385-418,1$ pl.

Markowski (S.). - Untersuchungen über die Helminthenfauna der Raben (Corvidæ) von Polen. Mém. Acad. Polon. Sc. et Lettres, Série B, 1933, n 5, p. 1-65.

Monishita (K.). - On the Trematodes of the genus Cyclocœlum obtained in Japan, with notes on the phylogeny of the Monostomous Trematodes. Dobutsu Gaku Zasshi, XXXVI, 1924, p. 231-234.

Nicoll (W.). - A reference list of the Trematode parasites of British Birds. Parasitology, XV, 1923, p. 151-202. 
Prudhoe (S.). - On some Trematodes from Ceylon. Ann. and Mag. Nat. Hist. (Sér. II), 1944, p. 1-13.

Ranliete (A.). - Trématodes hépatiques des Oiseaux, C.R. Soc. Biol., Série II, 1900 , p. 239-242.

RAYevsky (V. N.). - Renicola paraquinta n. sp. from Larus ridibundus. Papers on Helminthology published in commemoration of the 30 years jubileum of K. I. Skrjabin. Acad. Sc. de I'U.R.S.S., Moscou, 1937, p. 565-568.

Sinitsin (D.). - Studien über die Phylogenie der Trematoden. V. Revision of Harmostomina in the light of new facts from their morphology and life history. Zeitschr. für Parasitenkunde, III, 1931, p. 786-835.

SKrJabin (K. I.). - Nierentrematoden der Vögel Russlands. Centralbl. für Bakter. und Párasitenk, LXII, 1924, p. 80-90.

- Trematodes of animals and man. Elements of Trematodology. Ac. Sc. U.R.S.S., Moscou, 1947, 515 p., 220 fig.

Sokolowa-Andronowa (E. W.). - The renal Trematodes of birds of Far-West. Ac. Agricult. Moscou, 1937, p. 671-672.

Strom (J. K.). - On the fauna of Trematode worms from the wild animals of Kirghisia. Mag. Parasit. Moscou, VIII, 1940, p. 189-224 (en russe, résumé en anglais).

- Note on the classification of the Dicrocoliine. Mag. Parasitol. Ac. Sc. U.R.S.S., Moscou, VIII, 1940, p. 176-188.

Teixeira de Freitas (J. F.). - Revisão da familia Eucotylidæ Skrjabin. Mem. Inst. Oswaldo Cruz, XLIX, 1951, p. 33-123, 74 pl.

Trmon-David (J.). - Sur une nouvelle espèce de Renicola, Trématode parasite du rein des Laridés. Bull. Inst. Océanogr. Monaco, 1933, n 616, 16 p., 6 fig.

- Un Cyclocœlidé nouveau dans les sacs aériens de la Pie. Cyclocœlum (Pseudhyptiasmus) Dollfusi n. sp. Bull. Soc. Zool. Fr., LXXV, 1950, p. 243-246.

- Un Renicola nouveau chez la Pie. Renicola bretensis n. sp. Bull. Soc. Zool. Fr., LXXVII, 1953, p. 504-510, 1 pl.

Travassos (L.). - Revisão da familia Dicrocceliidæ Odhner 1910. Monogr. Inst. Oswaldo Cruz, 1944, $\mathrm{n}^{\circ} 2,357$ p., 124 pl.

Witenberg (G.). - Versuch eine Monographie der Trematodenfamilie Harmostominæ Braun. Zool. Jahrb. (System), LI, 1926, p. 167-254, 2 pl.

- Parasitische Würmer von Puffinus Kühlii. Ergebnisse der Sinä̈-Expedition 1927 der hebraischen Universitüt Jerusalem, 1929.

Yamagut (S.). - Studies on the Helminth fauna of Japan. Part. 25. Trematodes of Birds. IV. Jap. Journ. of Zoology, VIII, 1939, p. 191-192. 